\title{
Synchronization in the connectome: Metastable oscillatory modes emerge from interactions in the brain spacetime network
}

\author{
Joana Cabral 1,2,3*\#, Francesca Castaldo4\#, Jakub Vohryzek2,5, Vladimir Litvak ${ }^{4}$, Christian Bick 6,7,8,9, \\ Renaud Lambiotte ${ }^{8}$, Karl Friston ${ }^{4}$, Morten L. Kringelbach ${ }^{1,2,3,10}$, Gustavo Deco ${ }^{11,12}$
}

\author{
${ }^{1}$ Life and Health Sciences Research Institute, School of Medicine, University of Minho, Braga, Portugal \\ 2 Centre for Eudaimonia and Human Flourishing, Linacre College, University of Oxford, Oxford, United Kingdom \\ ${ }^{3}$ Center for Music in the Brain, Department of Clinical Medicine, Aarhus University, Aarhus, Denmark \\ ${ }^{4}$ Wellcome Centre for Human Neuroimaging, University College London, Queen Square Institute of Neurology, London, \\ United Kingdom \\ ${ }^{5}$ Center for Brain and Cognition, Computational Neuroscience Group, Universitat Pompeu Fabra, Barcelona, Spain \\ ${ }^{6}$ Department of Mathematics, Vrije Universiteit Amsterdam, Amsterdam, The Netherlands \\ ${ }^{7}$ Amsterdam Neuroscience - Systems \& Network Neuroscience, Amsterdam, The Netherlands \\ 8 Mathematical Institute, University of Oxford, Oxford, United Kingdom \\ ${ }^{9}$ Department of Mathematics, University of Exeter, Exeter, United Kingdom \\ 10 Department of Psychiatry, University of Oxford, Oxford, United Kingdom \\ ${ }^{11}$ Institució Catalana de la Recerca i Estudis Avançats (ICREA), Barcelona, Spain \\ 12 Department of Neuropsychology, Max Planck Institute for Human Cognitive and Brain Sciences, Germany
}

\# Shared first authorship

* Corresponding author:

joanacabral@med.uminho.pt

$+351919289649$ 


\section{Summary (150 words)}

1 A rich repertoire of oscillatory signals is detected from human brains with electro- and magnetoencephalography (EEG/MEG). However, the principles underwriting coherent oscillations and their link with neural activity remain unclear. Here, we hypothesise that the emergence of transient brain rhythms is a signature of weakly stable synchronization between spatially distributed brain areas, occurring at network-specific collective frequencies due to non-negligible conduction times. We test this hypothesis using a phenomenological network model to simulate interactions between neural mass potentials (resonating at $40 \mathrm{~Hz}$ ) in the structural connectome. Crucially, we identify a critical regime where metastable oscillatory modes emerge spontaneously in the delta $(0.5-4 \mathrm{~Hz})$, theta $(4-8 \mathrm{~Hz})$, alpha $(8-13 \mathrm{~Hz})$ and beta $(13-30 \mathrm{~Hz})$ frequency bands from weak synchronization of subsystems, closely approximating the MEG power spectra from 89 healthy individuals. Grounded in the physics of delay-coupled oscillators, these numerical analyses demonstrate the role of the spatiotemporal connectome in structuring brain activity in the frequency domain.

\section{Introduction}

The human brain is one of the most complex networks in nature, exhibiting a rich repertoire of activity patterns organized not only in space and time but also in the frequency domain. Indeed, rhythmicity is a central property of brain function - and perhaps of all biotic self organisation: from fast gamma activity in neurons to the life-cycle itself ${ }^{1-3}$. Within the broad range of oscillations emerging at frequencies between $0.05 \mathrm{~Hz}$ and $500 \mathrm{~Hz}$, the oscillations detected extracranially with electro- and magnetoencephalography (EEG/MEG) in resting humans typically peak between 0.5 and $30 \mathrm{~Hz}$, being categorized as delta $(\sim 0.5-4 \mathrm{~Hz})$, theta $(\sim 4-8 \mathrm{~Hz})$, alpha $(\sim 8-13 \mathrm{~Hz})$ and beta $(\sim 13-30 \mathrm{~Hz})^{4}$. topographies lasting up to a few hundred milliseconds ${ }^{5-7}$. 

oscillatory activity does not have a purely local origin and is associated to synchronization between distant neural assemblies ${ }^{8-12}$. Notably, there is a correlation between the distance over which synchronization is observed and the frequency of the synchronized oscillations $s^{13,14}$. Specific brain circuitries, including among others the thalamocortical loop, have been proposed to play a role in the generation of rhythmic activity ${ }^{15-17}$, which appears disrupted in neurological/neuropsychiatric disorders ${ }^{1,18}$. Still, the fundamental mechanisms driving the spontaneous emergence of short-lived spectrally resolved oscillatory patterns remain unclear ${ }^{8,19-21}$. time-delayed, which can significantly impact network activity in the frequency domain. Indeed, delay-coupled oscillators have been demonstrated to synchronize at frequencies slower than the natural frequency of the oscillators, leading to a form of collective frequency emerging from synchronization mechanisms ${ }^{22,23}$. Briefly, when $N$ phase oscillators - with natural frequency $\omega-$ are coupled together with a time delay $\tau$, they synchronize at a delay- and interaction-dependent collective frequency $\Omega$ given by $\Omega=\omega /\left(1+K^{*} N^{*} \tau\right)$, where $\mathrm{K}$ is the global coupling strength ${ }^{22}$. However, this phenomenon was demonstrated for networks of phase oscillators coupled to nearestneighbours with homogeneous delays ${ }^{22}$, and it is unclear how it generalizes to more complex realworld systems exhibiting collective rhythmicity, such as the brain. network structure of long axonal projections connecting brain areas - the so-called structural connectome $e^{24}$ - can shape brain activity in space and time ${ }^{25-33}$. Particularly, simulations of oscillatory units interacting in the connectome reveal a critical regime where different subsets of units temporarily synchronize and desynchronize, leading to transiently correlated activity across spatially segregated units ${ }^{27,34-36}$. This reinforces the hypothesis that long-range functional connectivity 
between brain areas is driven by synchronization mechanisms ${ }^{21,37-41}$. Importantly, when considering realistic time delays in simulations of phase oscillators coupled in the connectome, periods of increased synchrony are accompanied by increased power at slower frequencies, generating spatially-organized band-limited power fluctuations similar to the ones captured with MEG ${ }^{35}$. While these numerical results reveal the critical role of time delays to generate collective oscillations at reduced frequencies, the underlying mechanistic principle remains unclear. Further, it remains to be verified whether this phenomenon holds in the more realistic setting, wherein local oscillations have fluctuating amplitude, as observed empirically in electrophysiological recordings of neural $\operatorname{activity}^{42,43}$.

To address these fundamental questions, we build a phenomenological brain network model with realistic connectivity and time delays by representing each node as a Stuart-Landau oscillator operating in the underdamped regime in the presence of background noise $\mathrm{e}^{31,32,44,45}$. We set the natural frequency of all units in the gamma frequency range $(40 \mathrm{~Hz})$ and hypothesize that the power spectra of MEG recordings of healthy humans at rest will be approximated in a critical range of parameters where metastable synchronization occurs at reduced collective frequencies following analytic prediction.

\section{Results}

The reduced brain network model comprises $N=90$ dynamical units representing anatomically defined brain areas coupled according to the structural connectome with reciprocal (i.e., bidirectional/symmetric) coupling (Figure 1a). Each unit is described by a Stuart-Landau oscillator operating in the underdamped regime, such that when perturbed it decays to a fixed-point equilibrium with a damped oscillation at a natural frequency $\omega$ (Figure $1 \mathrm{~b}$, see Methods and 
bioRxiv preprint doi: https://doi.org/10.1101/2022.01.06.475196; this version posted January 6,2022 . The copyright holder for this preprint (which was not certified by peer review) is the author/funder, who has granted bioRxiv a license to display the preprint in perpetuity. It is made available under aCC-BY-NC-ND 4.0 International license.

a

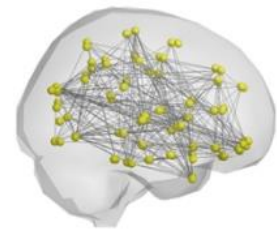

Dynamical units resonating at $40 \mathrm{~Hz}$

Weighted reciprocal coupling

Distance proportional to fibre length b

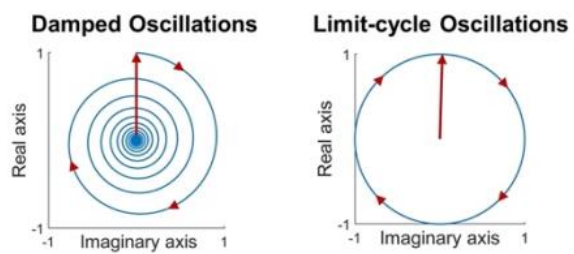

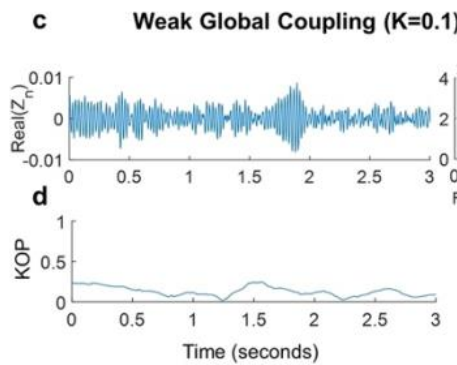

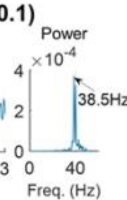

Intermediate Global Coupling (K=10)

Strong Global Coupling (K=50)
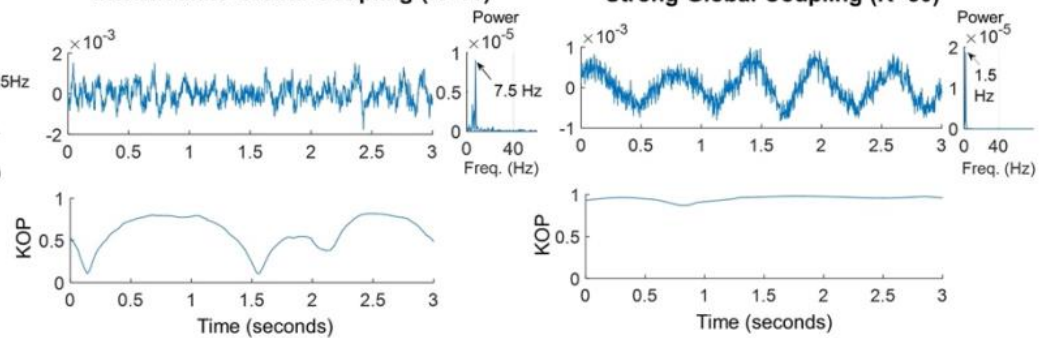

e
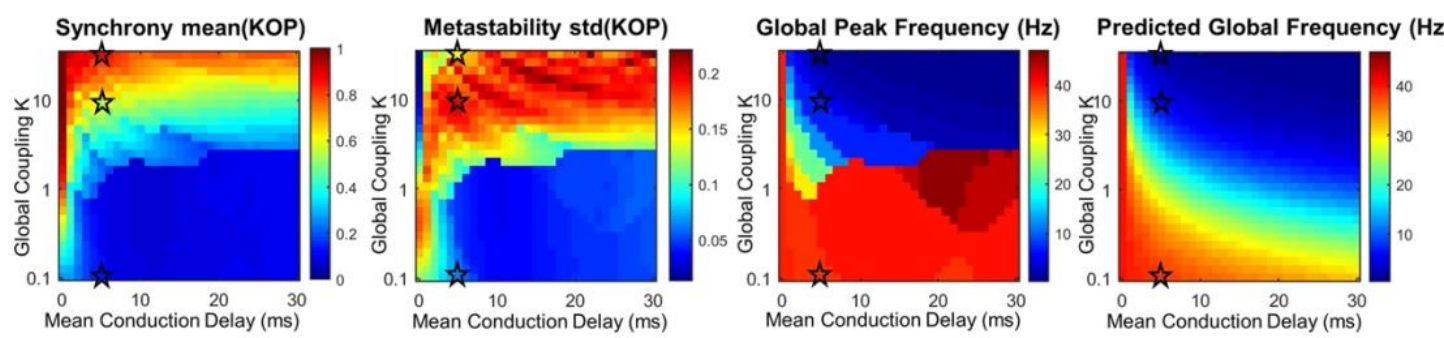

Figure 1 - Collective frequencies emerge from synchronization in the presence of delays. a-The phenomenological brain

network model consists in $N=90$ nodes representing anatomically defined brain regions with links representing axonal

projections between them. $\boldsymbol{b}$-A Stuart-Landau oscillator in the subcritical regime responding to perturbation (vertical

arrow) with an underdamped oscillation (left), in contrast with the super critical regime where the amplitude remains

constant (right). c-Simulated signal and corresponding power spectrum in a representative node obtained for three levels of global coupling, with a mean conduction delay of 5 milliseconds and in the presence of white noise. For weak coupling

(left) the simulated signal exhibits mostly noise-driven oscillations peaking close to the node's natural frequency. For

intermediate coupling (middle), weakly stable synchronization generates transient oscillations at reduced frequencies. For strong coupling (right), global synchronization becomes stable, and all units are entrained in a collective oscillation at a reduced frequency. $\boldsymbol{d}$ - The Kuramoto Order Parameter (KOP) evaluates the degree of phase synchronization of the system. For intermediate coupling, fluctuations in the order parameter are indicative of metastability. $\mathbf{e}$-Simulations were run for 50 seconds over a range of couplings and delays. For each simulation, we report the mean and standard deviation of the KOP (referred to as Synchrony and Metastability ${ }^{46}$ ) together with the peak frequency of the global signal (i.e., $\sum_{n=1}^{N} Z_{n}$ ). The synchronization frequency predicted analytically from $\Omega=\omega /\left(1+K^{*} N^{*}\langle\tau\rangle\right)$ is reported on the right panel, showing agreement with simulation results for sufficient synchrony. 
masses driven by feedback inhibition) and each unit is perturbed with uncorrelated white noise. The model - represented mathematically by a system of delay coupled differential equations - is solved numerically for two parameter ranges: the global coupling strength, $K$, which scales all pairwise connections; and the mean conduction delay, $\langle\tau\rangle$, which scales the time delays between units in proportion to the fibre lengths (Figure 1, see Methods for details).

The synchrony degree of the system, evaluated using the Kuramoto Order Parameter (KOP), is modulated by the global coupling strength $K$ : for weak coupling all units exhibit oscillations close to the natural frequency $\omega$ and for sufficiently strong coupling all units tend to synchronize at a global collective frequency $\Omega$, which, in the presence of time-delays, is different from the natural frequency $\omega$ (Figure 1c-d). In the critical range between incoherence and full synchrony, periods of weakly stable synchronization drive slow fluctuations in the KOP (Figure 1c-d, middle).

Observing the levels of synchrony and metastability across the range of parameters explored (Figure 1e), we find that the critical value of $\mathrm{K}$ above which the system can synchronize increases logarithmically with the mean delay, in line with analytic predictions for coupled oscillators with heterogeneous delays ${ }^{47}$ (see Supplementary Methods, Section II, Figure S5 ). When synchronization occurs in the presence of delays, we observe a dramatic decrease in the global peak frequency, closely approximating the analytic prediction given by $\Omega=\omega /\left(1+K^{*} N^{*}\langle\tau\rangle\right)$ (Figure 1e) (see Supplementary Methods, Section II, Figure S6). These findings serve to verify that the phenomenon of synchronization at reduced collective frequencies is not restricted to coupled phase oscillators and generalizes to units in the subcritical regime as long as damped oscillations emerge in response to external perturbation (see Supplementary Methods, Section II, Figure S7). Further, it demonstrates that the peak frequency of synchronization can be approximated analytically from global variables such as the natural frequency $\omega$, the number of units $N$, the coupling strength $K$, and the mean delay $\langle\tau\rangle$. 
bioRxiv preprint doi: https://doi.org/10.1101/2022.01.06.475196; this version posted January $6,2022$. The copyright holder for this preprint (which was not certified by peer review) is the author/funder, who has granted bioRxiv a license to display the preprint in perpetuity. It is made available under aCC-BY-NC-ND 4.0 International license.

Simulations reveal spectral features of human brain activity

One characteristic feature of MEG (and EEG) signals from healthy humans at rest is the

transient emergence of oscillations in the alpha frequency range ( $8-13 \mathrm{~Hz})$, resulting in a peak in the power spectrum whose prominence varies strongly across people (see Figure $2 a$ for the normalized power spectrum of MEG signals from 89 healthy young adults resting with eyes open from the

Human connectome Project open-source database; details in Methods section).

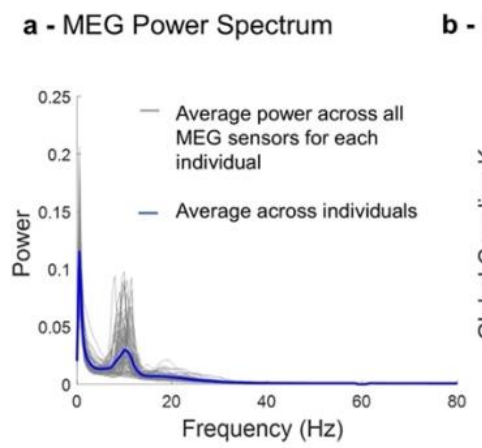

b - Disparity between MEG and Model PS

c-Model Power Spectra
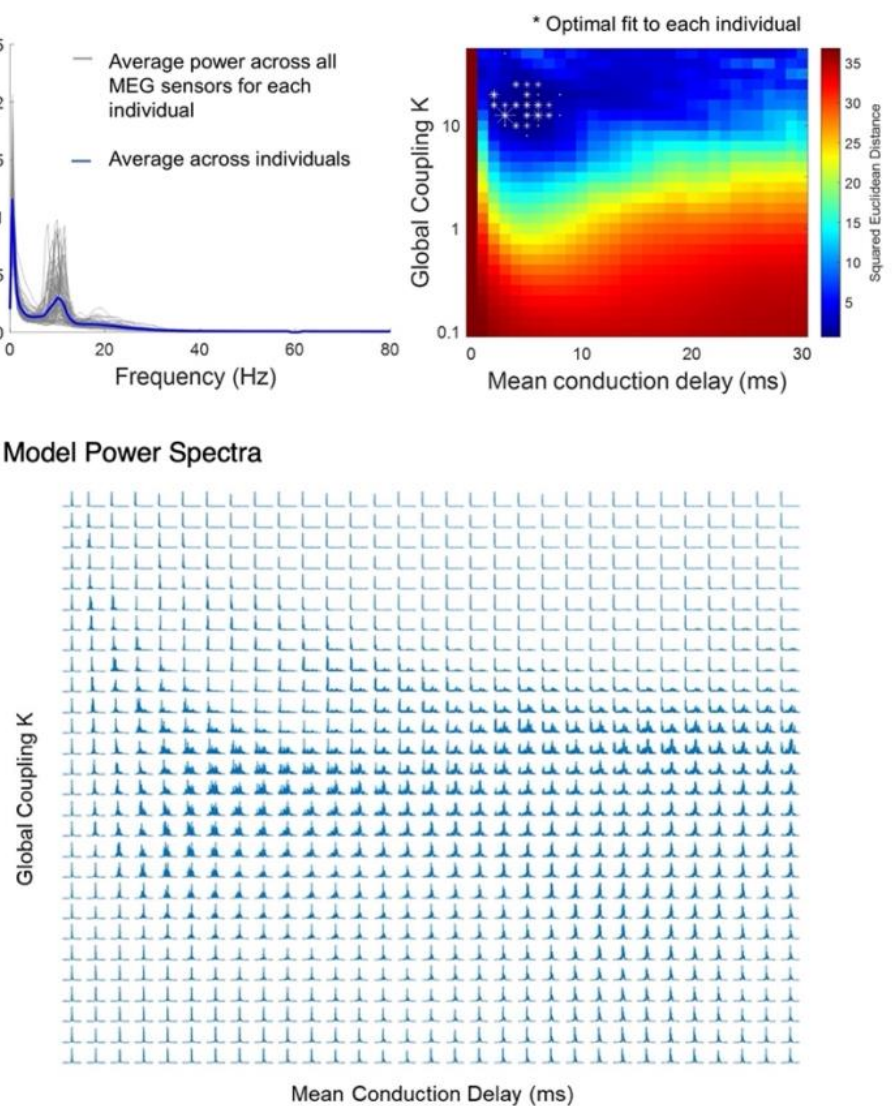

Figure 1 - The model approximates the MEG Power Spectrum (PS) from resting healthy human individuals in a critical

range of parameters. a - MEG power spectra from 89 healthy young adults resting with eyes open from the open-source

database of the Human connectome Project. The average power spectrum across individuals is reported in blue. $\boldsymbol{b}-$

Squared Euclidean distance between the MEG power spectrum averaged across all sensors and subjects and the power power spectra of each of the 89 individuals (size scaled according to the number of subjects in each point). c - For each pair 

resting subjects within the critical region of high metastability where synchronization occurs at reduced collective frequencies (comparing Figure $2 b$ with Figure $1 e$ ). In detail, for each pair of model parameters we calculate the squared Euclidean distance between the power spectrum of the approximates the individual MEG power spectra of each of the 89 participants, falling in 24 pairs of parameters (white asterisks in Figure 2b). Notably, this reveals a confined region in parameter space for a range of average delays $\langle\tau\rangle$ of 2 to 8 milliseconds, where slight changes in the coupling strength and conduction speed can account for individual differences in the MEG power spectra, with longer/shorter time delays and stronger/weaker coupling inducing shifts in the peak frequency and modulating the relative power at the peak frequency.

\section{Metastable Oscillatory Modes emerge from weakly stable cluster synchronization}

In the range of parameters where the model optimally approximates the power spectrum of

MEG signals, fluctuations in the magnitude of the order parameter are driven by metastable cluster synchronization. In other words, when the coupling is strong, but not sufficiently strong to stabilize

full synchronization, some subsets of units that are more strongly connected together (i.e., clusters/communities) can engage in partially-synchronized modes that remain stable for a short period in time. Given the presence of time delays, these clusters do not synchronize at the natural frequency of the individual units $(40 \mathrm{~Hz})$, but instead synchronize at slower cluster-specific collective frequencies, leading to the emergence of metastable oscillatory modes (MOMs) at sub-gamma frequencies. 
bioRxiv preprint doi: https://doi.org/10.1101/2022.01.06.475196; this version posted January 6, 2022. The copyright holder for this preprint (which was not certified by peer review) is the author/funder, who has granted bioRxiv a license to display the preprint in perpetuity. It is made available under aCC-BY-NC-ND 4.0 International license.

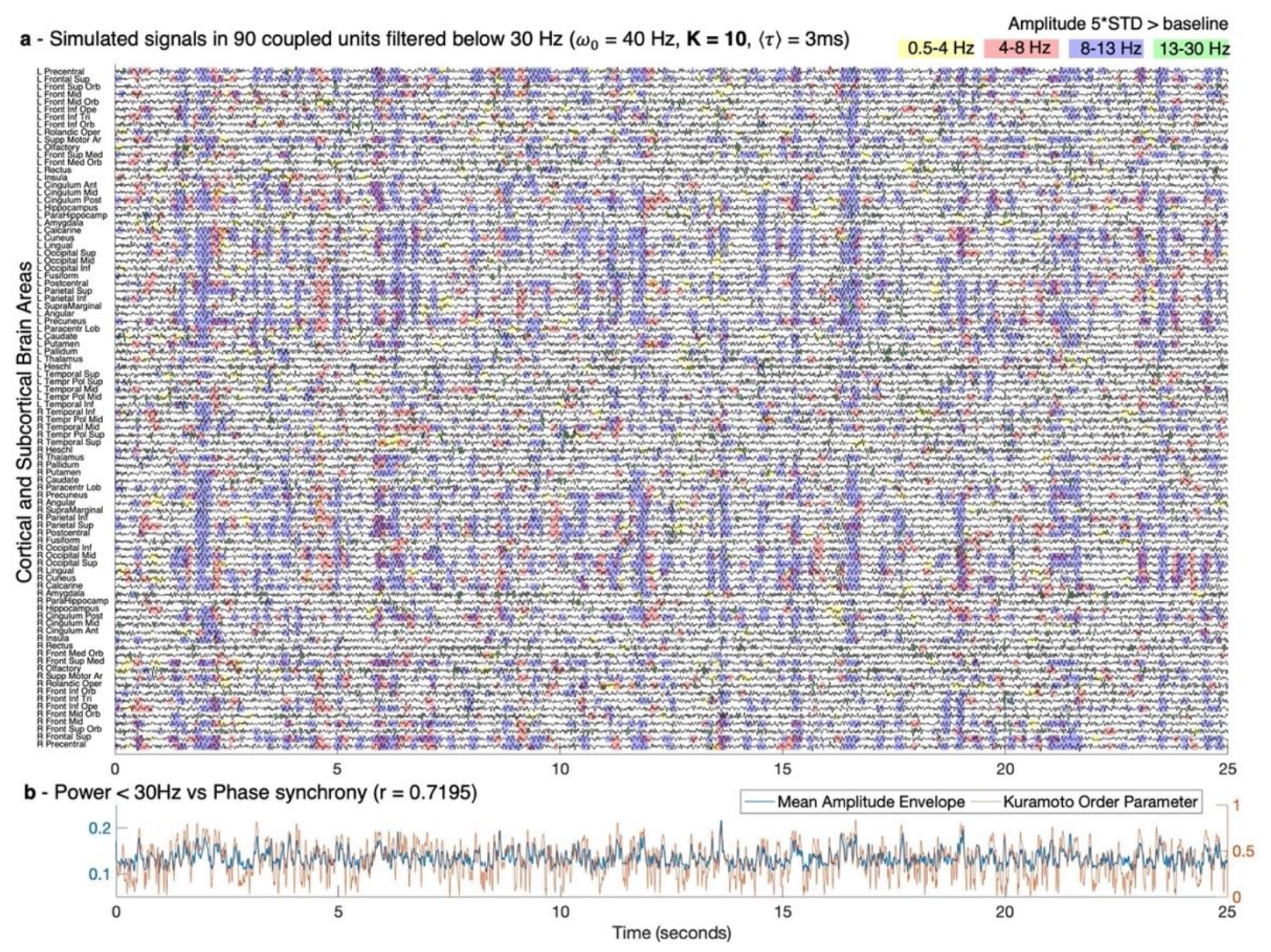

Figure 3. Sub-gamma oscillations emerge from weakly stable cluster synchronization. a-An example of the simulated

signals in all 90 units plotted over 25 seconds, each representing a brain area from the AAL brain parcellation template, points of increased power in the delta (yellow), theta (red), alpha (blue) and beta (green) frequency bands. For each frequency band, the threshold was defined as 5 standard deviations (STD) of the amplitude - in the same frequency bands when no delays were considered. $\boldsymbol{b}$-The mean amplitude envelope (blue) of the filtered signals shown in panel A correlates with $r=0.7195$ with the phase synchronization evaluated by the Kuramoto Order Parameter (orange, right $y$-axis). 
bioRxiv preprint doi: https://doi.org/10.1101/2022.01.06.475196; this version posted January 6, 2022. The copyright holder for this preprint (which was not certified by peer review) is the author/funder, who has granted bioRxiv a license to display the preprint in perpetuity. It is made available under aCC-BY-NC-ND 4.0 International license.

164 for the alpha frequency range in Figure 3a. Notably, for different sets of parameters, the configuration of Figure 3a changes strongly. Indeed, while for very weak coupling, almost no supra-

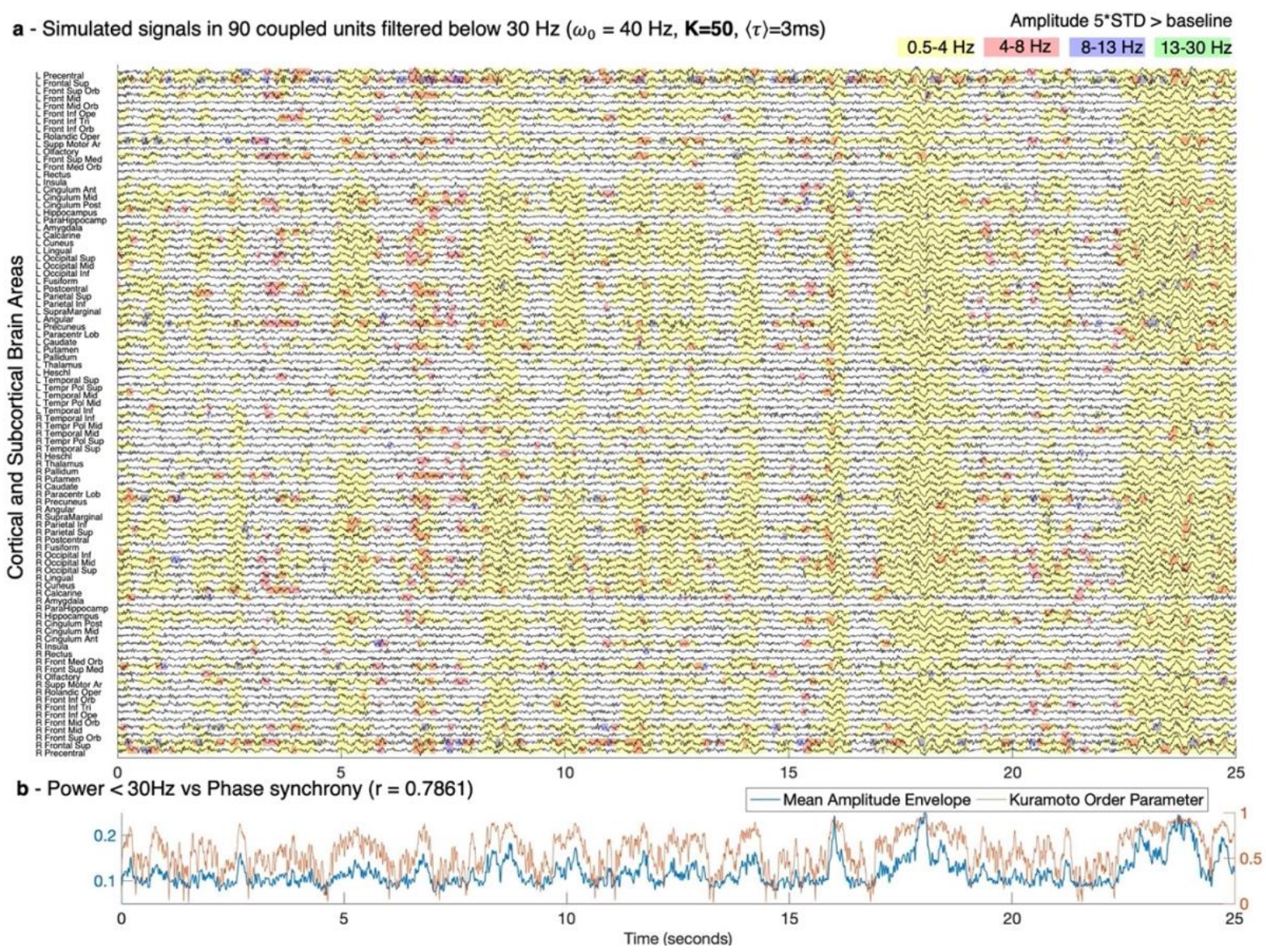

Figure 4 - Global delta waves emerge for strong coupling. a-The simulated signals in all 90 units plotted over 25 seconds, oscillatory activity typically detected with MEG. Shades highlight the time points of increased power in the delta (yellow), theta (red), alpha (blue) and beta (green) frequency bands. For each frequency band, the threshold was defined as 5 

parameters is illustrated in Supplementary Video VS1, where the emergence of supra-threshold oscillations is represented over time in the brain for different sets of parameters.

Furthermore, the power at sub-gamma frequencies is found to correlate strongly with the instantaneous phase synchronization evaluated by the KOP over time $(r=0.7195$ and $r=0.7861$ for Figure $3 \mathrm{~b}$ and Figure $4 \mathrm{~b}$ correspondingly). This demonstrates that the emergence of oscillations at sub-gamma frequencies in the simulations is modulated by fluctuations in the synchrony degree. frequency bands for different sets of model parameters in terms of their duration (i.e., consecutive time that the power remains above threshold), their size (i.e., the number of units simultaneous displaying power above threshold) and occupancy (i.e., the proportion of time that the power is detected above threshold) (Figure 5). As can be seen, in the range of parameters where optimal fits to MEG data are obtained (central panel), the alpha MOMs last longer, recruit more units and occur more often. Importantly, we demonstrate that the global parameters such as the coupling strength and the conduction speed can modulate the spatiotemporospectral properties of the whole system in a non-trivial way, while the dynamics at the local level and the underlying structural network remain unchanged. 


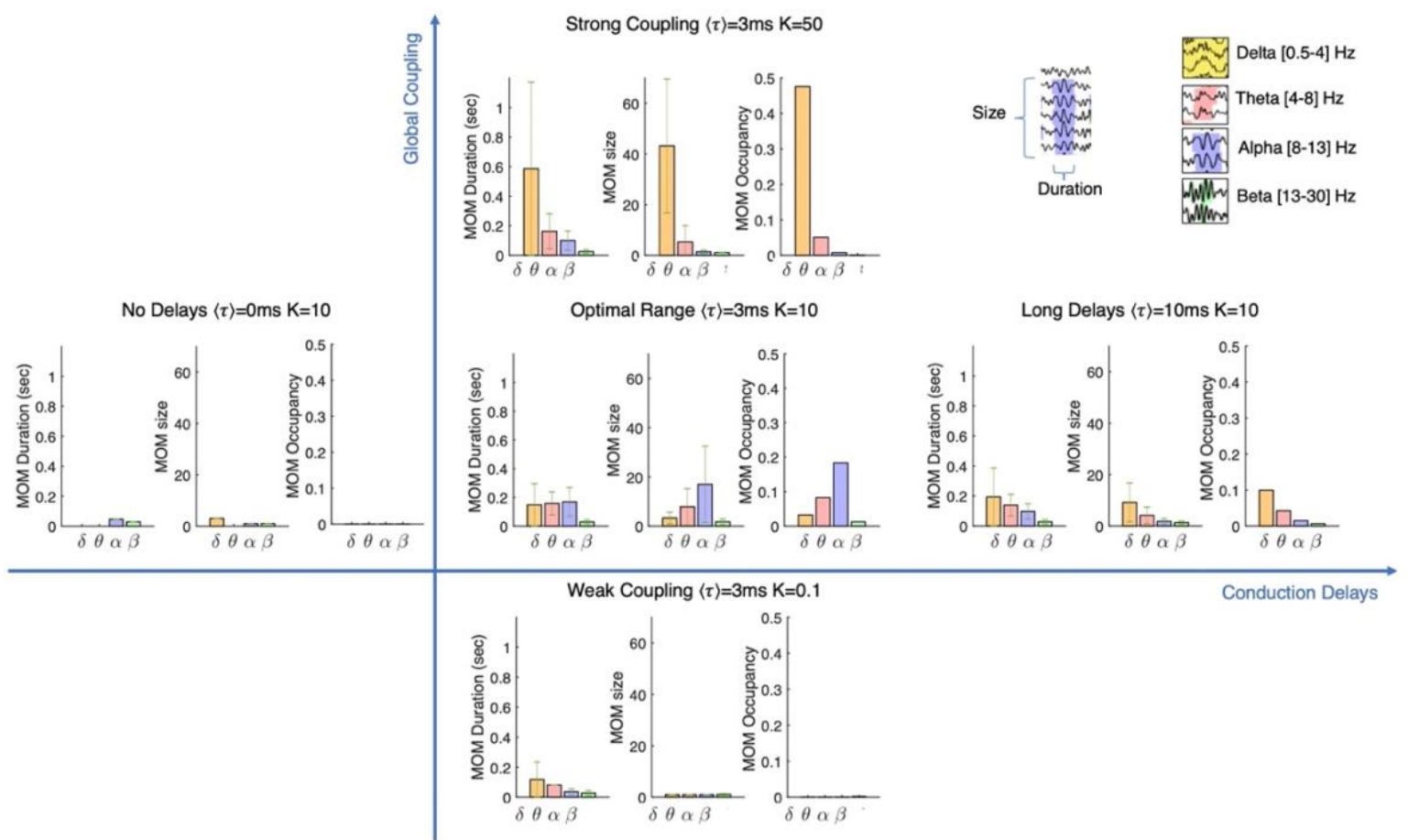

194

Figure 5. Characterization of metastable oscillatory modes (MOMs) emerging from the system for different sets of parameters. MOMs are characterized in terms of duration (i.e., consecutive time that the power remains above threshold), size (i.e., the number of units simultaneous displaying power above threshold) and occupancy (i.e., the proportion of time that the power is detected above threshold over the entire simulation), for each frequency band. This demonstrates that the same network structure, i.e., the connectome, can exhibit different oscillatory modes organized in space and in time, depending on global parameters of the system, namely the Global Coupling strength and the Conduction Delays (which are inversely proportional to the conduction speed). In the critical range of parameters (central plot), oscillations in the alpha frequency band emerge more frequently and involve more units. Notably, globally synchronized delta oscillations - as typically observed in states of reduced consciousness - are associated to an increase in the global coupling strength. See also Supplementary Video VS1.

Spatial patterns of frequency-specific envelope covariance Finally, we investigate the spatial patterns of connectivity by computing the covariance matrices of the envelopes in each frequency band (Figure 6a) and count the components with eigenvalue (i.e., associated power) above the largest eigenvalue of the same band-specific envelope covariance matrices obtained in the case of no delays (Figure 6b). For weak coupling, the envelope covariance matrices are mostly random in all frequency bands considered and no meaningful component is detected. In the optimal range, the covariance matrices reveal frequency-specific 
214 illustrates how, given the same underlying spacetime structure (i.e., the matrices of coupling weights

a
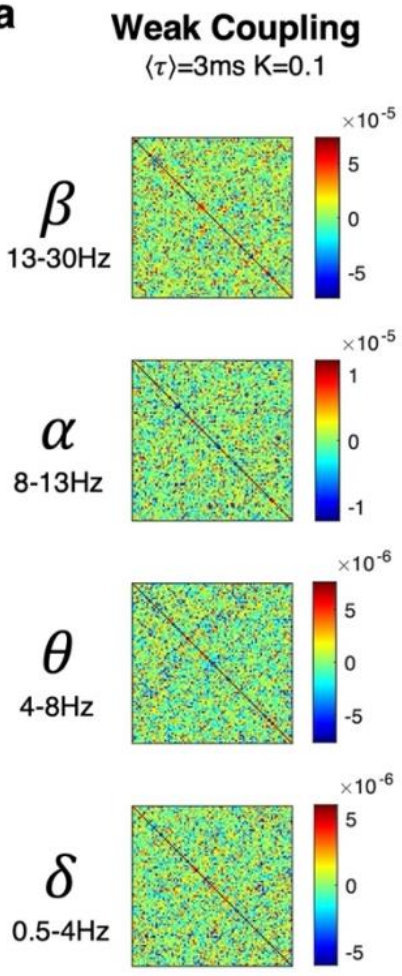

b

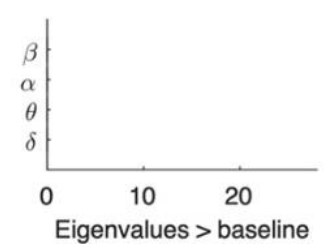

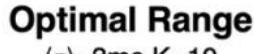

$\langle\tau\rangle=3 \mathrm{~ms} \mathrm{~K}=10$
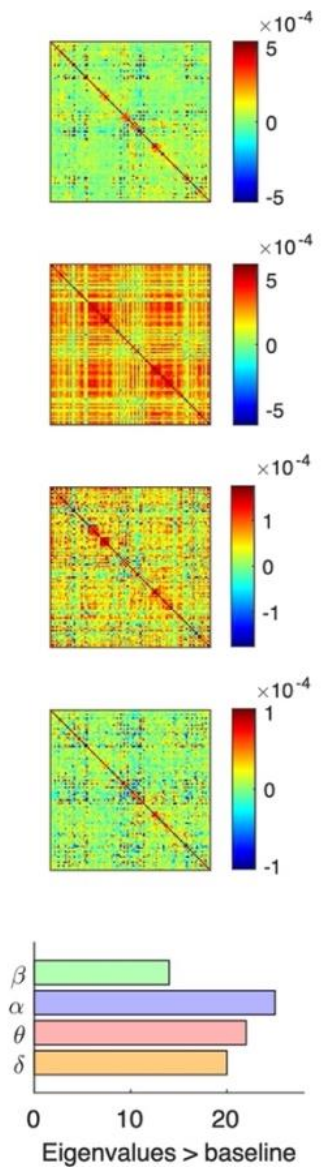

\section{Strong Coupling \\ $\langle\tau\rangle=3 \mathrm{~ms} \mathrm{~K}=50$}
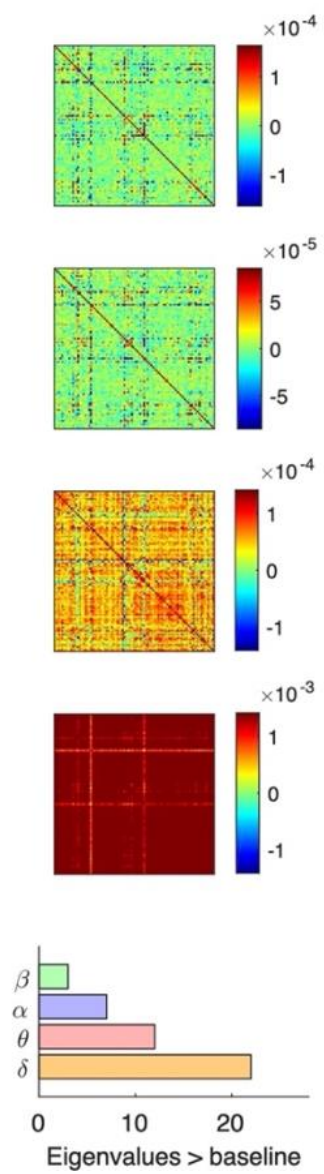

\section{Long Delays \\ $\langle\tau\rangle=10 \mathrm{~ms} \mathrm{~K}=10$}
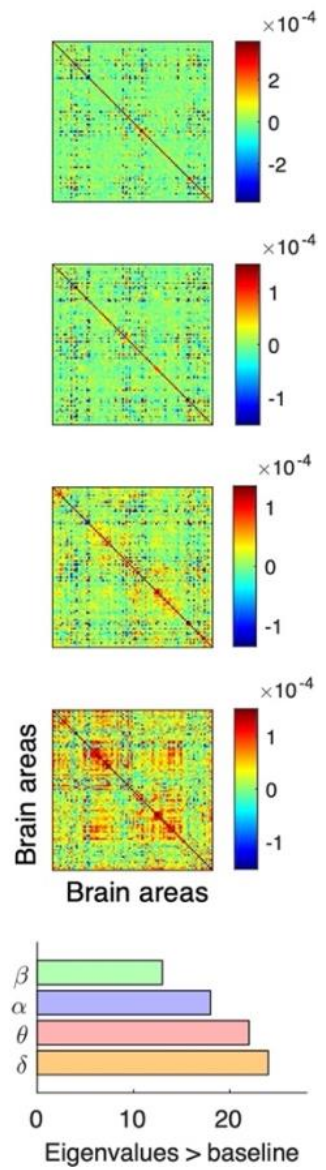

Figure 6. Repertoire of frequency-specific envelope covariance patterns emerging from synchronization in the 


\section{Discussion}

In nature, an extraordinary variety of life processes result from the coordination of

interacting dynamical systems, where the collective ensemble engages in functionally-relevant

activity patterns that cannot be inferred from the isolated system alone ${ }^{48-51}$. Progress in biophysics

implies uncovering the physical mechanisms underlying life processes, grounding the inner

complexity of biological systems to universal theoretical principles ${ }^{52,53}$.

Here, we describe a universal synchronization mechanism occurring in networks with a

complex spacetime structure (i.e., with heterogeneous connectivity and finite conduction speeds)

driving the emergence of metastable oscillatory modes at reduced collective frequencies. We

propose this mechanism underwrites the formation of macroscopic spatiotemporally organized

oscillatory signals recorded with EEG/MEG. Our hypothesis is endorsed using a phenomenological

brain network model, reduced to its key essential ingredients to allow efficient numerical

approximations to analytic predictions, but at the same time sufficiently complex to allow a fair

approximation of MEG spectral features.

The deliberate reductionist perspective inherent in this brain network model is intended to

link with theoretical works on delay-coupled oscillatory systems $22,47,54,55$. Towards this end, we

consider only $\mathrm{N}=90$ identical units with same natural frequency, same bifurcation parameter and

same noise level coupled in the structural connectome. Therefore, we focus solely on the effects of

global variables, namely the global coupling $K$ and the mean conduction delay $\langle\tau\rangle$ in the emerging

synchronization phenomena. To establish the construct validity of our numerical simulations, we

show that the peak synchronization frequency can be approximated by the analytic prediction

derived for synthetic networks of coupled Kuramoto oscillators with time delays ${ }^{22}$. Further, in line

with theoretical predictions ${ }^{47,56}$, we find that the complex spacetime topology of the structural 
provides a robust framework to test a theoretically grounded mechanistic scenario for the spontaneous formation of frequency-specific long-range coherence in complex networks.

the model, namely by adding more units, tuning the intrinsic frequencies and bifurcation parameter of each brain area, or modulating the level of input noise to mimic local modulatory effects, in order to improve the fitting to empirical data from individuals in different conditions. Further, given the potential universality of this synchronization mechanism, we predict that it can be extended to more realistic biophysical models of neural networks ${ }^{57}$, providing a mechanistic interpretation for some of the self-organizing phenomena emerging in systems for which a precise analytic prediction cannot be solved. spectrum of coupled oscillatory systems. Although the frequency of the oscillations considered herein is relatively fast with respect to the ultra-slow fluctuations $(<0.1 \mathrm{~Hz})$ detected with functional Magnetic Resonance Imaging (fMRI), it is important to highlight that metastable synchronization drives power fluctuations on ultra-slow timescales, and therefore, even small time delays can significantly modulate spontaneous activity at ultra-slow time-scales. Importantly, we note that for the model to exhibit the predicted behaviour, the integration step for numerical simulations needs to be sufficiently small (here the simulation results were found to stabilize for $\mathrm{dt} \leq 10^{-4}$ seconds), which significantly increases the computation time (here each simulation of 50 seconds lasted approximately 5 hours). 

approximates healthy awake brain activity, we find that higher coupling enhances global order, where the whole brain displays slow coherent oscillations in the delta-range $(0.5-2 \mathrm{~Hz})$, which nicely approximate the most powerful brain rhythms detected during unconscious states such as slowwave sleep, coma or anaesthesia. On the other hand, operating at weaker coupling hinders the formation of MOMs at sub-gamma frequencies, altering the spectral profile similarly to what is observed in M/EEG recordings of patients with neuropsychiatric disorders associated to disconnection, such as schizophrenia, where the power in alpha appears to be significantly reduced $^{60-63}$. Such abnormal interactions within cortico-subcortical oscillatory networks may emerge from specific local deregulation or neural circuit disruption ${ }^{64}$. However, how a local change may alter the communication between brain-areas and brain network dynamics remains an open question. Overall, these results are aligned with recent works proposing that spontaneous transitions between multiple space-time patterns on complex networks provide a solid theoretical framework for the interpretation of the non-stationary but recurrent macroscopic patterns emerging spontaneously in brain activity, and ultimately supporting brain function ${ }^{65,66}$. From a technical perspective, it may be surprising that this kind of itinerant dynamics emerges under symmetrical coupling between nodes; in the sense that asymmetric coupling is normally required for breaking detailed balance - and engendering stochastic chaos of the sort described above. However, the dynamics of each node are generated with asymmetric Jacobians, suggesting that symmetry breaking of intrinsic connectivity is a sufficient condition for the nonequilibrium dynamics that characterise real brains. support cognitive functions remains unclear ${ }^{21,59,67-69}$. One possibility is that the areas engaged in a MOM are directly involved in long-range functional integration, but another is that these areas are inhibited by entering in a collective low-energy mode ${ }^{12,38}$. Shedding some light on this open question, we find that synchronization with delays induces not only a shift to slower frequencies but 
with distributed delays ${ }^{70}$ (see the vertical axes in Figure 1C). From a 'metabolic' perspective, this shows that MOMs can be approached as 'low energy modes' with respect to high power gamma oscillations, providing a physical explanation for the emergence of the so-called 'idle rhythms ${ }^{171}$. brain stimulation.

\section{Materials and Methods}

\section{Ethics statement}

All human data used in this study is from the public repository of the Human connectome Project

$(\mathrm{HCP})^{72}$ (https://www.humanconnectome.org), which is distributed in compliance with international ethical guidelines.

\section{Structural connectome}

The $N x N$ matrices of structural connectivity, C, and distances, D, used in the brain network model were computed from diffusion spectrum and T2-weighted Magnetic Resonance Imaging (MRI) data obtained from 32 participants scanned at the Massachusetts General Hospital centre for the Human connectome Project ${ }^{73}$ (http://www.humanconnectome.org/).

Briefly, the data were processed using a generalized q-sampling imaging algorithm implemented in DSI Studio (http://dsi-studio.labsolver.org). Segmentation of the T2-weighted anatomical images produced a white-matter mask and co-registering of the images to the b0 image of the diffusion data using SPM12. In each participant, 200,000 fibres were sampled within the white-matter mask. Fibres were transformed into Montreal Neurological Institute (MNI) space using Lead-DBS876. 
The connectivity matrix $\mathrm{C}$ was obtained by counting the number of fibres detected between each pair of $N=90$ brain areas defined in the Automated Anatomical Labelling (AAL) parcellation scheme ${ }^{74}$.

Similarly, the distance matrix D was obtained by computing the mean length of all fibres detected between each pair of $N=90$ brain areas (see Supplementary Methods section V, Figure S9).

\section{Neuroimaging acquisition and pre-processing}

We used the human resting-state MEG data publicly available from the Human connectome Project (HCP) consortium, acquired on a Magnes 3600 MEG (4D Neurolmaging) with 248 magnetometers. male, acquired in three subsequent sessions lasting 6 min each).

A set of pre-processing pipelines are run on all MEG data prior to further resting-state analysis. To measure the power spectrum of the resting state MEG data at the sensor level, we used the "powavg" pipeline. The continuous data are segmented, Hanning-tapered, Fourier-transformed and the estimated power is averaged over all segments. The power spectrum is represented as FieldTrip structure in a MATLAB file. Notch filters are then applied to the band-pass filtered data to remove the power line noise (cut-off frequencies $59-61 \mathrm{~Hz}$ and $119-121 \mathrm{~Hz}$ ). All the details are extracted and further explained in the HCP reference manual

ference Manual.pdf).

\section{Brain Network Model}

The Stuart-Landau (SL) equation (Equation 1) is the canonical form to describe the behaviour of a system near an Andronov-Hopf bifurcation, i.e. exhibiting the birth of an oscillation from a fixed point ${ }^{39,75}$. In other words, it is used to describe systems that have a static fixed point (like a resting spring), but respond to perturbation with an oscillation, which may damped or self-sustained 
Methods, Figure Sx). This model allows to designate complex-systems behaviour among several applications, bridging the gap between the simplicity of the Kuramoto model and the extensiveness of the phase-amplitude frameworks ${ }^{76,77}$. It describes how the oscillator behaves both when it is weakly attracted to a limit cycle (displaying only damped oscillations in response to perturbation) and, on the other hand, when it is purely restricted to a limit cycle (oscillations remain selfsustained).

Our analysis is based on a system of $\mathrm{N}=90 \mathrm{SL}$ oscillators coupled in the connectome, considering both the connectivity strength, $C_{n p}$, and the conduction delays, $\tau_{n p}$, between each pair of brain areas $n$ and $p$. The conduction delays are defined in proportion to the fiber lengths between brain areas, assuming an homogenous condtuction speed $\mathrm{v}$, such that $\tau_{n p}=D_{n p} / v$, where $D_{n p}$ is the length of the fibres detected between brain areas $n$ and $p$. To simulate how the activity in node $n$ is affected by the behaviour of all other nodes $p(p \in N \wedge p \neq n)$, we describe the interaction between nodes in the form:

$$
\frac{d Z_{n}}{d t}=Z_{n}\left[a+i \omega-\left|Z_{n}^{2}\right|\right]+K \sum_{p \neq n}^{N} C_{n p}\left[Z_{p}\left(t-\tau_{n p}\right)-Z_{n}(t)\right]+\beta \eta_{1}+i \beta \eta_{2}, \forall n \in N
$$

where the complex variable $Z_{n}(t)$ describes the state of the $n^{\text {th }}$ oscillator at time $t$. The first term in equation (1) describes the intrinsic dynamics of each unit, the second term describes the input received from coupled units and the last terms represent uncorrelated white noise (see Supplementary Methods, Sections I and II for detailed analysis of the model).

With this approach, we consider that the first term of Eq. 1 represents the natural excitability of neuronal assemblies, where $\omega=2 \pi * f_{f}$ is the angular frequency, with $f_{f}$ as the fundamental frequency. For our proof of concept, we set all nodes with identical natural frequency $\omega_{0}=2 \pi *$ $40 \mathrm{~Hz}$, representing the undifferentiated ability of a neural mass to engage in gamma-frequency oscillations. 
The parameter $a$ determines the position of the each unit with respect to the limit cycle. For $a>0$, a stable limit cycle appears via a superciritical Hopf bifurcation, while when $a<0$ there is only a stable fixed point at the origin $Z_{n}=0$, so the bifurcation point is at $a=0$. Importantly, if $a$ is sufficiently close to the bifurcation, the system is still weakly attracted to the limit cycle and damped oscillations emerge in response to external input, with a decay time scaled by $a$. In this work, we pick a value of $a=-5$ for all nodes, such that a single input drives a damped oscillation decaying after $\sim 1 s$, approximating the slowest decay time-constants of inhibitory receptors (Figure S4) (tdecay $(G A B A B) \approx 500-1000 m s)$. In Supplementary Methods (section II, Figure S7) we show that our results are qualitatively similar for a broad range of $a$ values, both positive and negative, thus demonstrating the generalizability of synchronization at collective frequencies to coupled oscillatory systems with fluctuating amplitude, be they damped or self-sustained. We note that this mechanism only fails when the units have an overdamped response (exponential decay without oscillation), which, in this case, only occurred for $a=-500$. Thus it is of great interest in future research to investigate whether the local bifurcation parameters can be tuned based on sensitive observables to fit the MEG data of different individuals in different conditions.

The second term represents the total input received from other brain areas, scaled by parameter $K$, which sets the strength of all network interactions with respect to the intrinsic node dynamics.

Because we wish to focus on the nonlinear phenomena introduced by time delays, we model the node-to-node interactions using a particular linear diffusive coupling, as the simplest approximation of the general coupling function, considering delayed interactions. Here, the signal of node $n$ at time $t$ is calculated with respect to the activity of all other nodes $p$ at time $t-\tau_{n p}$ (where $\tau_{n p}$ is the time delay between $n$ and $p$ ), scaled by the relative coupling strength given by $C_{n p}$.

The third term of Equation 1 represents the real and imaginary part of Gaussian noise. In this analysis, the system is perturbed with uncorrelated white noise, where $\eta_{1}$ and $\eta_{2}$ are independently drawn from a Gaussian distribution with mean zero and standard deviation $\beta=0.001$. 
387 from the MEG data we aim at explaining). For a qualitative comparison with the literature in delay-

seconds with an integration step $d t=10^{-4}$ seconds.

\section{Kuramoto Order Parameter}

To evaluate the global synchrony of the simulated network activity over time, we use the Kuramoto

order parameter:

$$
R(t) e^{i \Theta(t)}=\frac{1}{N} \sum_{n=1}^{N} e^{i \theta_{n}(t)}
$$

where $\theta_{n}(t)$ is the phase of each node, given by the argument of $Z_{n}$. The temporal evolution of the

Order Parameter $R(t)$ provides an instantaneous measure of the degree of synchrony of the

ensemble. Since we add noise in the simulations, we first band-pass filter the signals $Z_{n}$ around the asynchronous, $\langle R(t)\rangle^{\sim} 0$ or synchronous $\left.\langle R(t)\rangle \sim 1\right)$, whereas fluctuations in the order parameter are indicative of Metastability, driven by constant transitions between different weakly stable solutions ${ }^{57}$. For the analysis in parameter space, we take the mean $\langle R(t)\rangle$ as a measure of the global synchronization while the standard deviation $S T D(R(t))$ indicates how much $R(t)$ fluctuates in time $^{46}$. 


\section{Analytic Prediction of Collective Frequency of Synchronization}

$$
\dot{\theta}_{n}(t)=\omega+K \sum_{p \neq n}^{N} C_{n p} \sin \left[\theta_{p}\left(t-\tau_{n p}\right)-\theta_{n}(t)\right]
$$

406

407

\section{Model Performance}

414 We perform a parameter space exploration by tuning the two free parameters $K$ and $\langle\tau\rangle$. We choose to increase $K$ exponentially as a power of 10 from $10^{-1}$ to $10^{1.7}$ in steps of $10^{0.1}$, to ensure a range that covers from weak to strong coupling. $\langle\tau\rangle$ is explored in the range from $0 \mathrm{~ms}$ to $30 \mathrm{~ms}$ in

417 steps of $1 \mathrm{~ms}$.

418 We measure the fitting between the empirical sensor MEG PS for each of the 89 subjects and the 419 simulated PS for each pair of parameters as the squared Euclidean distance, resulting in one fitting 
value for each subject. This can be regarded as a maximum likelihood procedure under the assumption of Gaussian observation noise.

\section{Metastable Oscillatory Modes}

To detect MOMs and characterize them in space and time, we band-pass filter the simulated signals

in each frequency band and obtain the corresponding amplitude envelopes using the Hilbert

427 Since some areas are more coupled together than others, even with "zero delays" these areas exhibit more power in those frequencies that is purely due to noisy interactions. Therefore, we define a different threshold for each node and each band.

\section{Envelope Covariance Patterns}

To detect the covariance patterns for each frequency band we calculate the NxN covariance

baseline case - that is null delays and intermediate coupling.

\section{Author contributions}

435 Conceptualization: JC, MLK, GD

436 Methodology: JC, JV, KF, GD

437 Investigation: JC, FC

438 Visualization: JC, FC

439 Funding acquisition: JC, VL, MLK, GD

440 Project administration: JC, GD

441 Supervision: VL, KF, MLK, GD

442 Writing - original draft: JC, FC

443 Writing - review \& editing: JC, FC, JV, CB, RL, KF, VL, MLK, GD 


\section{Competing interests:}

Authors declare that they have no competing interests.

Data and materials availability:

\section{Acknowledgments}

$\mathrm{JC}$ is funded by the Portuguese Foundation for Science and Technology grants Human data were provided by the Human connectome Project, WU-Minn Consortium (Principal

All simulations and analysis were performed in Matlab. The codes and materials used in this study are available in: https://liveuclac-my.sharepoint.com/:f:/g/personal/skgtfca ucl ac uk/Er9J06K5bxCoqaAzRRIIp4BSTm7 LWGzNWKoKROXgLJig?e=uiWIHA

which will be deposited in a public database upon acceptance. UIDB/50026/2020,UIDP/50026/2020 and CEECIND/03325/2017. FC is funded by the EU-project euSNN European School of Network Neuroscience (MSCA-ITN-ETN H2020-860563). The Wellcome Centre for Human Neuroimaging is supported by core funding from Wellcome [203147/Z/16/Z]. JV is supported by the EU H2020 FET Proactive project Neurotwin grant agreement no. 101017716. RL acknowledges support from EPRSC Grants No. EP/V013068/1 and EP/V03474X/1. MLK is supported by the Center for Music in the Brain, funded by the Danish National Research Foundation (DNRF117), and the Centre for Eudaimonia and Human Flourishing, funded by the Pettit Foundation and Carlsberg Foundation. GD is supported by the Spanish national research project (PID2019105772GB-I00 MCIU AEI), funded by the Spanish Ministry of Science, Innovation and Universities (MCIU), State Research Agency (AEI); HBP SGA3 Human Brain Project Specific grant agreement 3 (945539), funded by the EU H2020 FET Flagship program; SGR Research Support Group (reference 2017 SGR 1545), funded by the Catalan Agency for Management of University and Research Grants (AGAUR); Neurotwin Digital twins for model-driven non-invasive electrical brain stimulation (grant agreement 101017716), funded by the EU H2020 FET Proactive program; euSNN (grant agreement 860563), funded by the EU H2020 MSCA-ITN Innovative Training Networks; The Emerging Human Brain Cluster (CECH) (001-P-001682) within the framework of the European Research Development Fund Operational Program of Catalonia 2014-2020; Brain-Connects: Brain Connectivity during Stroke Recovery and Rehabilitation (201725.33), funded by the Fundacio La Marato TV3; and Corticity, FLAG-ERA JTC 2017 (reference PCI2018-092891), funded by the MCIU, AEI. Investigators: David Van Essen and Kamil Ugurbil; 1U54MH091657) funded by the $16 \mathrm{NIH}$ Institutes and Centers that support the NIH Blueprint for Neuroscience Research; and by the McDonnell Center for Systems Neuroscience at Washington University. 


\section{References}

4741 Uhlhaas, P. J. \& Singer, W. Neural synchrony in brain disorders: relevance for cognitive dysfunctions and pathophysiology. Neuron 52, 155-168 (2006).

2 Engel, A. K., Fries, P. \& Singer, W. Dynamic predictions: oscillations and synchrony in topdown processing. Nature reviews. Neuroscience 2, 704-716, doi:10.1038/35094565 (2001).

3 Buzsáki, G. \& Draguhn, A. Neuronal oscillations in cortical networks. Science 304, 1926-1929 (2004).

4 Buzsaki, G. Rhythms of the Brain. (Oxford University Press, 2006).

5 Baker, A. P. et al. Fast transient networks in spontaneous human brain activity. elife 3, e01867, doi:10.7554/eLife.01867 (2014).

6 Vidaurre, D. et al. Spectrally resolved fast transient brain states in electrophysiological data. Neurolmage 126, 81-95, doi:10.1016/j.neuroimage.2015.11.047 (2016).

7 Michel, C. M. \& Koenig, T. EEG microstates as a tool for studying the temporal dynamics of whole-brain neuronal networks: a review. Neurolmage 180, 577-593 (2018).

8 Jensen, O., Spaak, E. \& Zumer, J. M. in Magnetoencephalography 359-403 (Springer, 2014).

9 Sherman, M. A. et al. Neural mechanisms of transient neocortical beta rhythms: Converging evidence from humans, computational modeling, monkeys, and mice. Proceedings of the National Academy of Sciences 113, E4885-E4894 (2016).

10 Varela, F., Lachaux, J.-P., Rodriguez, E. \& Martinerie, J. The brainweb: phase synchronization and large-scale integration. Nature reviews neuroscience 2, 229 (2001).

11 Hipp, J. F., Hawellek, D. J., Corbetta, M., Siegel, M. \& Engel, A. K. Large-scale cortical correlation structure of spontaneous oscillatory activity. Nature neuroscience 15, 884-890, doi:10.1038/nn.3101 (2012).

12 Palva, S. \& Palva, J. M. Discovering oscillatory interaction networks with M/EEG: challenges and breakthroughs. Trends in cognitive sciences 16, 219-230 (2012).

13 Schnitzler, A. \& Gross, J. Normal and pathological oscillatory communication in the brain. Nature reviews neuroscience 6, 285-296 (2005).

14 Von Stein, A. \& Sarnthein, J. Different frequencies for different scales of cortical integration: from local gamma to long range alpha/theta synchronization. International journal of psychophysiology 38, 301-313 (2000).

15 Bhattacharya, S., Cauchois, M. B., Iglesias, P. A. \& Chen, Z. S. The impact of a closed-loop thalamocortical model on the spatiotemporal dynamics of cortical and thalamic traveling waves. Scientific reports 11, 1-19 (2021).

16 Freyer, F. et al. Biophysical mechanisms of multistability in resting-state cortical rhythms. The Journal of neuroscience : the official journal of the Society for Neuroscience 31, 63536361, doi:10.1523/JNEUROSCI.6693-10.2011 (2011).

17 Cabral, J. et al. Exploring mechanisms of spontaneous functional connectivity in MEG: How delayed network interactions lead to structured amplitude envelopes of band-pass filtered oscillations. Neurolmage 90, 423-435, doi:10.1016/j.neuroimage.2013.11.047 (2014).

18 Llinás, R. R., Ribary, U., Jeanmonod, D., Kronberg, E. \& Mitra, P. P. Thalamocortical dysrhythmia: a neurological and neuropsychiatric syndrome characterized by magnetoencephalography. Proceedings of the National Academy of Sciences 96, 1522215227 (1999).

19 Vidaurre, D. et al. Spontaneous cortical activity transiently organises into frequency specific phase-coupling networks. Nature communications 9, 1-13 (2018).

20 O'Neill, G. C. et al. Dynamics of large-scale electrophysiological networks: A technical review. Neurolmage 180, 559-576 (2018).

21 Friston, K. J. Transients, metastability, and neuronal dynamics. Neurolmage 5, 164-171 (1997). 
22 Niebur, E., Schuster, H. G. \& Kammen, D. M. Collective frequencies and metastability in networks of limit-cycle oscillators with time delay. Physical review letters 67, 2753-2756, doi:10.1103/PhysRevLett.67.2753 (1991).

23 Atay, F. M., Jost, J. \& Wende, A. Delays, connection topology, and synchronization of coupled chaotic maps. Physical review letters 92, 144101, doi:10.1103/PhysRevLett.92.144101 (2004).

24 Sporns, O., Tononi, G. \& Kotter, R. The human connectome: A structural description of the human brain. PLoS computational biology 1, e42, doi:10.1371/journal.pcbi.0010042 (2005).

25 Honey, C. J., Kotter, R., Breakspear, M. \& Sporns, O. Network structure of cerebral cortex shapes functional connectivity on multiple time scales. Proceedings of the National Academy of Sciences of the United States of America 104, 10240-10245, doi:10.1073/pnas.0701519104 (2007).

26 Ghosh, A., Rho, Y., McIntosh, A. R., Kotter, R. \& Jirsa, V. K. Cortical network dynamics with time delays reveals functional connectivity in the resting brain. Cognitive neurodynamics $\mathbf{2}$, 115-120, doi:10.1007/s11571-008-9044-2 (2008).

27 Deco, G., Jirsa, V., McIntosh, A. R., Sporns, O. \& Kotter, R. Key role of coupling, delay, and noise in resting brain fluctuations. Proceedings of the National Academy of Sciences of the United States of America 106, 10302-10307, doi:10.1073/pnas.0901831106 (2009).

28 Cabral, J., Hugues, E., Sporns, O. \& Deco, G. Role of local network oscillations in resting-state functional connectivity. Neurolmage 57, 130-139, doi:10.1016/j.neuroimage.2011.04.010 (2011).

29 Cabral, J., Kringelbach, M. \& Deco, G. Functional Connectivity dynamically evolves on multiple time-scales over a static Structural Connectome: Models and Mechanisms. Neurolmage 160, 84-96, doi:10.1016/j.neuroimage.2017.03.045 (2017).

30 Deco, G. \& Kringelbach, M. L. Turbulent-like dynamics in the human brain. Cell reports 33, 108471 (2020).

31 Deco, G. et al. Single or Multi-Frequency Generators in on-going brain activity: a mechanistic whole-brain model of empirical MEG data. Neurolmage, doi:10.1016/j.neuroimage.2017.03.023 (2017).

32 Deco, G., Kringelbach, M. L., Jirsa, V. K. \& Ritter, P. The dynamics of resting fluctuations in the brain: metastability and its dynamical cortical core. Scientific reports 7, 3095, doi:10.1038/s41598-017-03073-5 (2017).

33 Tewarie, P. et al. How do spatially distinct frequency specific MEG networks emerge from one underlying structural connectome? The role of the structural eigenmodes. Neurolmage 186, 211-220 (2019).

34 Cabral, J., Hugues, E., Sporns, O. \& Deco, G. Role of local network oscillations in resting-state functional connectivity. Neurolmage 57, 130-139, doi:10.1016/j.neuroimage.2011.04.010 (2011).

35 Cabral, J. et al. Exploring mechanisms of spontaneous functional connectivity in MEG: how delayed network interactions lead to structured amplitude envelopes of band-pass filtered oscillations. Neurolmage 90, 423-435, doi:10.1016/j.neuroimage.2013.11.047 (2014).

36 Roberts, J. A. et al. Metastable brain waves. Nature communications 10, 1-17 (2019).

37 Ponce-Alvarez, A. et al. Resting-state temporal synchronization networks emerge from connectivity topology and heterogeneity. PLoS computational biology 11, e1004100, doi:10.1371/journal.pcbi.1004100 (2015).

38 Engel, A. K., Gerloff, C., Hilgetag, C. C. \& Nolte, G. Intrinsic coupling modes: multiscale interactions in ongoing brain activity. Neuron 80, 867-886 (2013).

39 Cocchi, L., Gollo, L. L., Zalesky, A. \& Breakspear, M. Criticality in the brain: A synthesis of neurobiology, models and cognition. Progress in neurobiology 158, 132-152 (2017).

40 Deco, G. et al. Rare long-range cortical connections enhance human information processing. Current Biology 31, 4436-4448. e4435 (2021). 
41 Brookes, M. J. et al. Measuring functional connectivity using MEG: methodology and comparison with fcMRI. Neurolmage 56, 1082-1104, doi:10.1016/j.neuroimage.2011.02.054 (2011).

42 Buhl, E. H., Tamas, G. \& Fisahn, A. Cholinergic activation and tonic excitation induce persistent gamma oscillations in mouse somatosensory cortex in vitro. The Journal of physiology $\mathbf{5 1 3}$ ( Pt 1), 117-126 (1998).

43 Sanchez-Vives, M. V. \& McCormick, D. A. Cellular and network mechanisms of rhythmic recurrent activity in neocortex. Nature neuroscience 3, 1027 (2000).

44 Samanta, H. S., Bhattacharjee, J. K., Bhattacharyay, A. \& Chakraborty, S. On noise induced poincaré-andronov-Hopf bifurcation. Chaos: An Interdisciplinary Journal of Nonlinear Science 24, 043122 (2014).

45 Selivanov, A. A. et al. Adaptive synchronization in delay-coupled networks of Stuart-Landau oscillators. Physical Review E 85, 016201 (2012).

46 Shanahan, M. Metastable chimera states in community-structured oscillator networks. Chaos 20, 013108, doi:10.1063/1.3305451 (2010).

47 Lee, W. S., Ott, E. \& Antonsen, T. M. Large coupled oscillator systems with heterogeneous interaction delays. Physical review letters 103, 044101 (2009).

48 Winfree, A. T. Biological rhythms and the behavior of populations of coupled oscillators. Journal of theoretical biology 16, 15-42 (1967).

49 Haken, H. Information and Self-Organization - A Macroscopic approach to Complex Systems. (Springer, 1988).

50 Mirollo, R. E. \& Strogatz, S. H. Synchronization of pulse-coupled biological oscillators. SIAM Journal on Applied Mathematics 50, 1645-1662 (1990).

51 Strogatz, S. H. \& Stewart, I. Coupled oscillators and biological synchronization. Scientific American 269, 102-109 (1993).

52 Pikovsky, A., Kurths, J., Rosenblum, M. \& Kurths, J. Synchronization: a universal concept in nonlinear sciences. (Cambridge university press, 2003).

53 Strogatz, S. Sync: The emerging science of spontaneous order. (Penguin UK, 2004).

54 Yeung, M. K. S. \& Strogatz, S. H. Time Delay in the Kuramoto Model of Coupled Oscillators. Physical review letters 82, 648-651 (1999).

55 Earl, M. G. \& Strogatz, S. H. Synchronization in oscillator networks with delayed coupling: A stability criterion. Physical Review E 67, 036204 (2003).

56 Wildie, M. \& Shanahan, M. Metastability and chimera states in modular delay and pulsecoupled oscillator networks. Chaos 22, 043131, doi:10.1063/1.4766592 (2012).

57 Bick, C., Goodfellow, M., Laing, C. R. \& Martens, E. A. Understanding the dynamics of biological and neural oscillator networks through exact mean-field reductions: a review. The Journal of Mathematical Neuroscience 10, 1-43 (2020).

58 Tononi, G., Sporns, O. \& Edelman, G. M. A measure for brain complexity: relating functional segregation and integration in the nervous system. Proceedings of the National Academy of Sciences 91, 5033-5037 (1994).

59 Tognoli, E. \& Kelso, J. A. The metastable brain. Neuron 81, 35-48, doi:10.1016/j.neuron.2013.12.022 (2014).

60 Cabral, J., Kringelbach, M. L. \& Deco, G. Functional graph alterations in schizophrenia: a result from a global anatomic decoupling? Pharmacopsychiatry 45 Suppl 1, S57-64, doi:10.1055/s-0032-1309001 (2012).

61 Cabral, J., Hugues, E., Kringelbach, M. L. \& Deco, G. Modeling the outcome of structural disconnection on resting-state functional connectivity. Neurolmage 62, 1342-1353, doi:10.1016/j.neuroimage.2012.06.007 (2012).

62 Schartner, M. M., Carhart-Harris, R. L., Barrett, A. B., Seth, A. K. \& Muthukumaraswamy, S. D. Increased spontaneous MEG signal diversity for psychoactive doses of ketamine, LSD and psilocybin. Scientific reports 7, 46421 (2017). 
$62563 \quad$ Carhart-Harris, R. L. et al. The entropic brain: a theory of conscious states informed by neuroimaging research with psychedelic drugs. Frontiers in human neuroscience 8, 20, doi:10.3389/fnhum.2014.00020 (2014).

64 Goriely, A., Kuhl, E. \& Bick, C. Neuronal oscillations on evolving networks: dynamics, damage, degradation, decline, dementia, and death. Physical review letters 125, 128102 (2020). Bick, C. Heteroclinic switching between chimeras. Physical Review E 97, 050201 (2018). Ansmann, G., Lehnertz, K. \& Feudel, U. Self-induced switchings between multiple space-time patterns on complex networks of excitable units. Physical Review X 6, 011030 (2016). Singer, W. Synchronization of cortical activity and its putative role in information processing

67 Singer, W. Synchronization of cortical activity and its putat doi:10.1146/annurev.ph.55.030193.002025 (1993).

68 Rabinovich, M. I., Simmons, A. N. \& Varona, P. Dynamical bridge between brain and mind. Trends in cognitive sciences 19, 453-461 (2015).

69 Roberts, J. A. et al. Metastable brain waves. Nature communications 10, 1056 (2019).

63970 Atay, F. M. Distributed delays facilitate amplitude death of coupled oscillators. Physical review letters 91, 094101, doi:10.1103/PhysRevLett.91.094101 (2003). inversely related to $\mathrm{fMRI-BOLD}$ signal in primary somatosensory and motor cortex. Human brain mapping 30, 1168-1187 (2009).

72 Van Essen, D. C. et al. The WU-Minn Human Connectome Project: an overview. Neurolmage 80, 62-79, doi:10.1016/j.neuroimage.2013.05.041 (2013). Setsompop, K. et al. Pushing the limits of in vivo diffusion MRI for the Human Connectome Project. Neurolmage 80, 220-233 (2013).

Tzourio-Mazoyer, N. et al. Automated anatomical labeling of activations in SPM using a macroscopic anatomical parcellation of the MNI MRI single-subject brain. Neurolmage 15,

$65175 \quad$ A., A. A., Vitt, A. A. \& Khakin, S. E. Theory of oscillators. Dover Mathematics (1987).

65276 Aranson, I. S. \& Kramer, L. The world of the complex Ginzburg-Landau equation. Reviews of Modern Physics 74, 99-143, doi:10.1103/RevModPhys.74.99 (2002). Olinger, D. J. A low-dimensional model for chaos in open fluid flows. Physics of Fluids A: Fluid Dynamics 5, 1947-1951, doi:10.1063/1.858821 (1993).

78 Sethia, G. C., Sen, A. \& Atay, F. M. Clustered chimera states in delay-coupled oscillator systems. Physical review letters 100, 144102, doi:10.1103/PhysRevLett.100.144102 (2008). 


\section{a - Simulated signals in 90 coupled units filtered below $30 \mathrm{~Hz}\left(\omega_{0}=40 \mathrm{~Hz}, \mathrm{~K}=10,\langle\tau\rangle=3 \mathrm{~ms}\right)$}

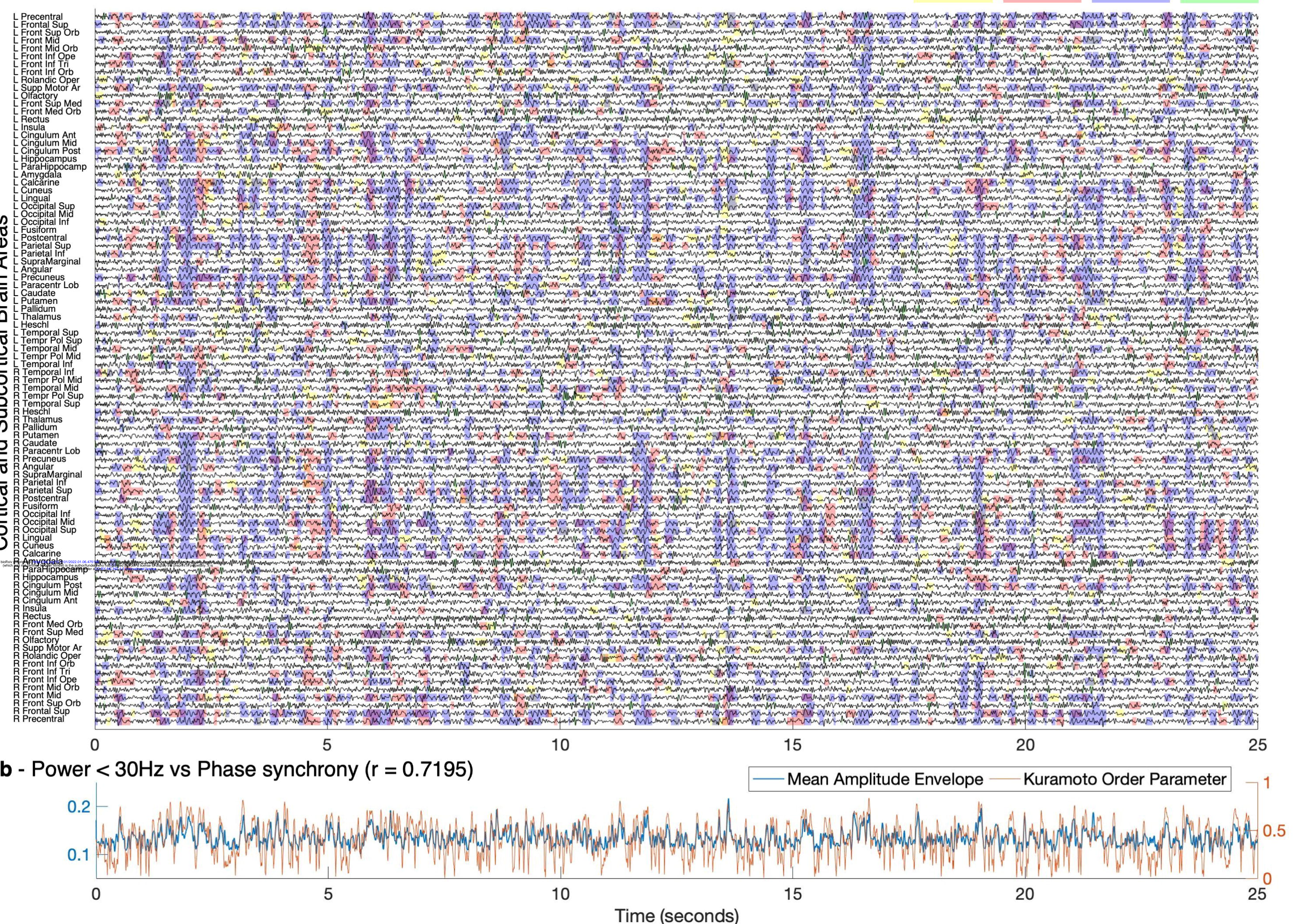




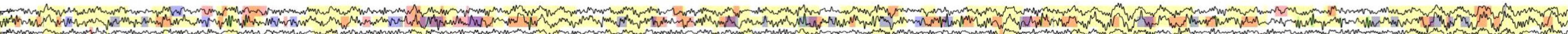

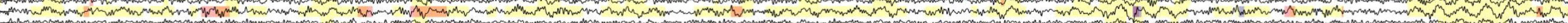
mom

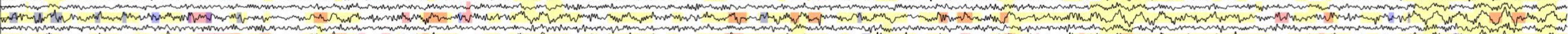

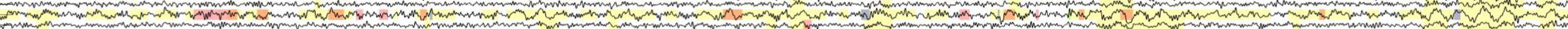
2xum

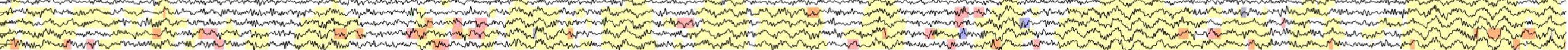

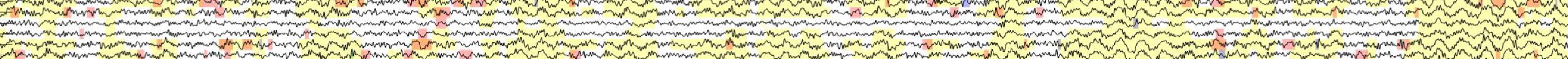

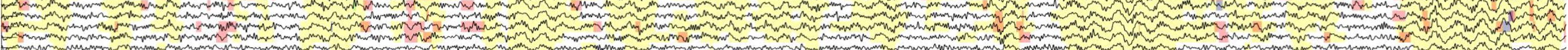

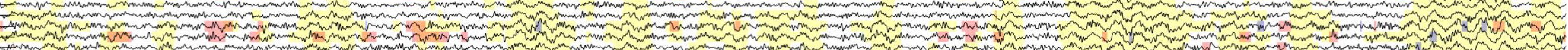
$T_{0}$ anm

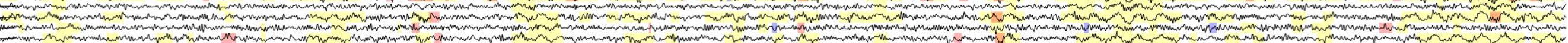

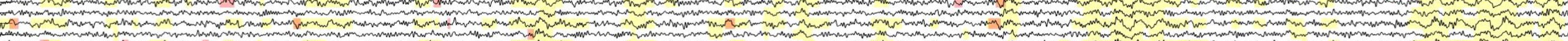
Tum

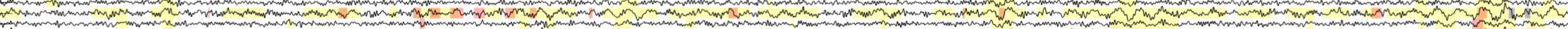
Dom

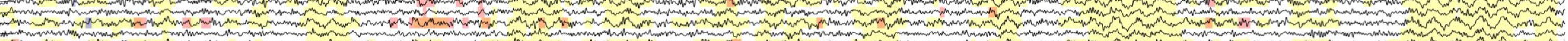

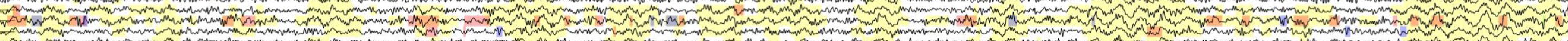

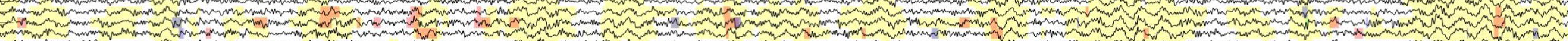

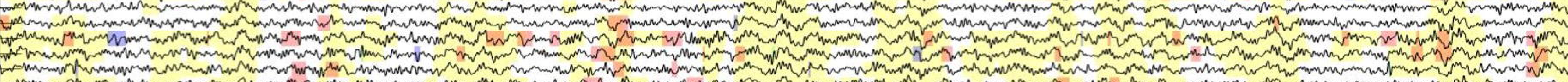
- $\rightarrow=0$ - mum 20

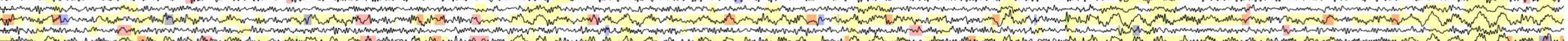
mon

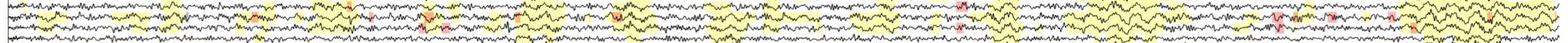

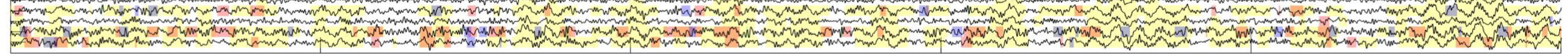
0 $5 \quad 10$ 15 20

b - Power $<30 \mathrm{~Hz}$ vs Phase synchrony $(r=0.7861)$ - Mean Amplitude Envelope Kuramoto Order Parameter

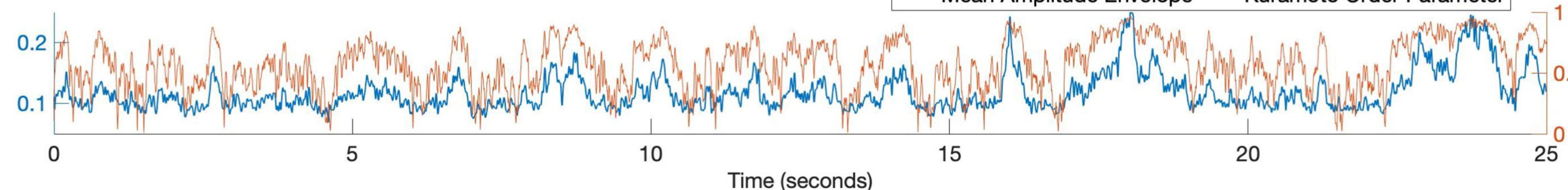


Strong Coupling $\langle\tau\rangle=3 \mathrm{~ms} \mathrm{~K}=50$

No Delays $\langle\tau\rangle=0 \mathrm{~ms} \mathrm{~K}=10$

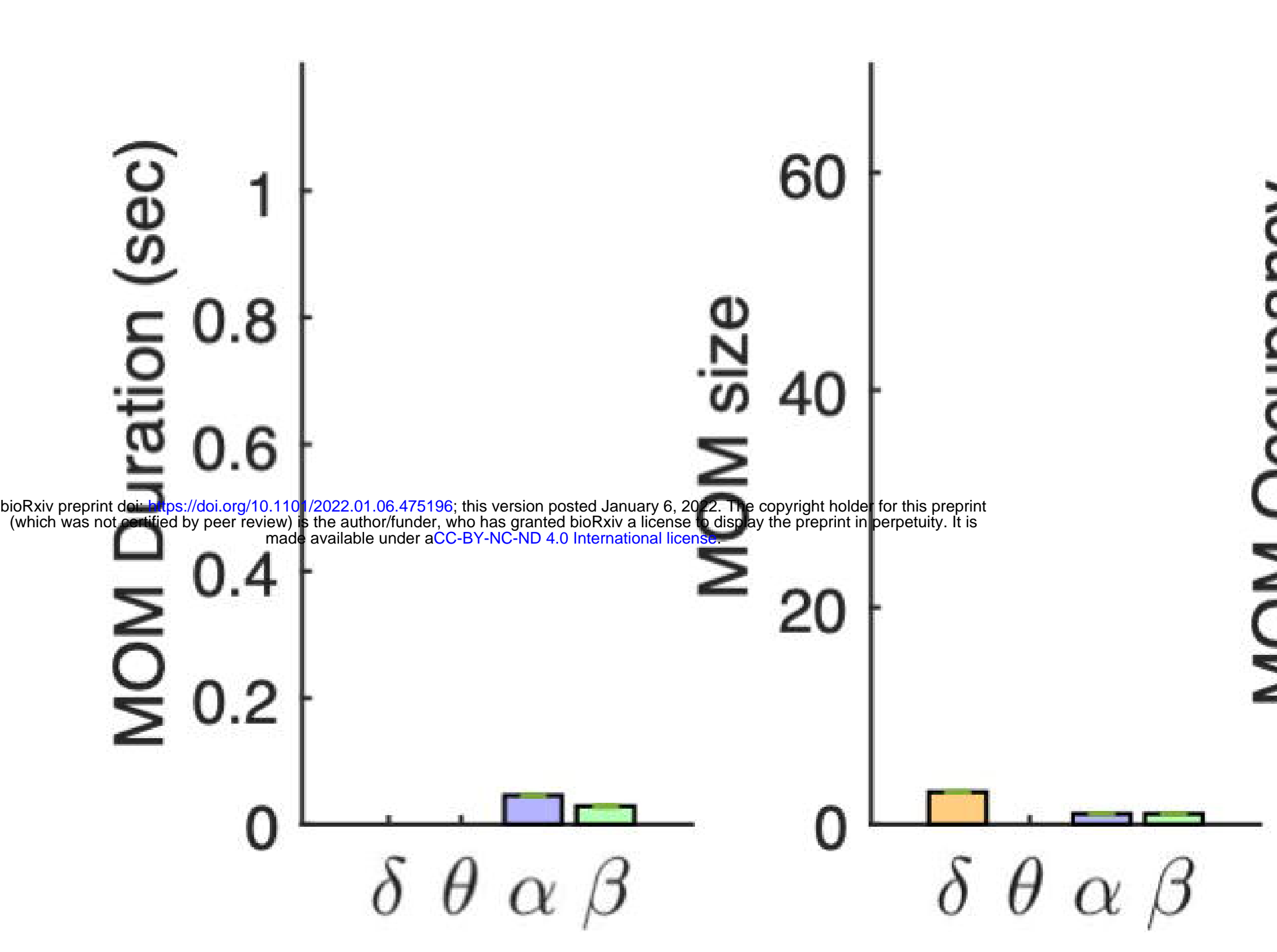

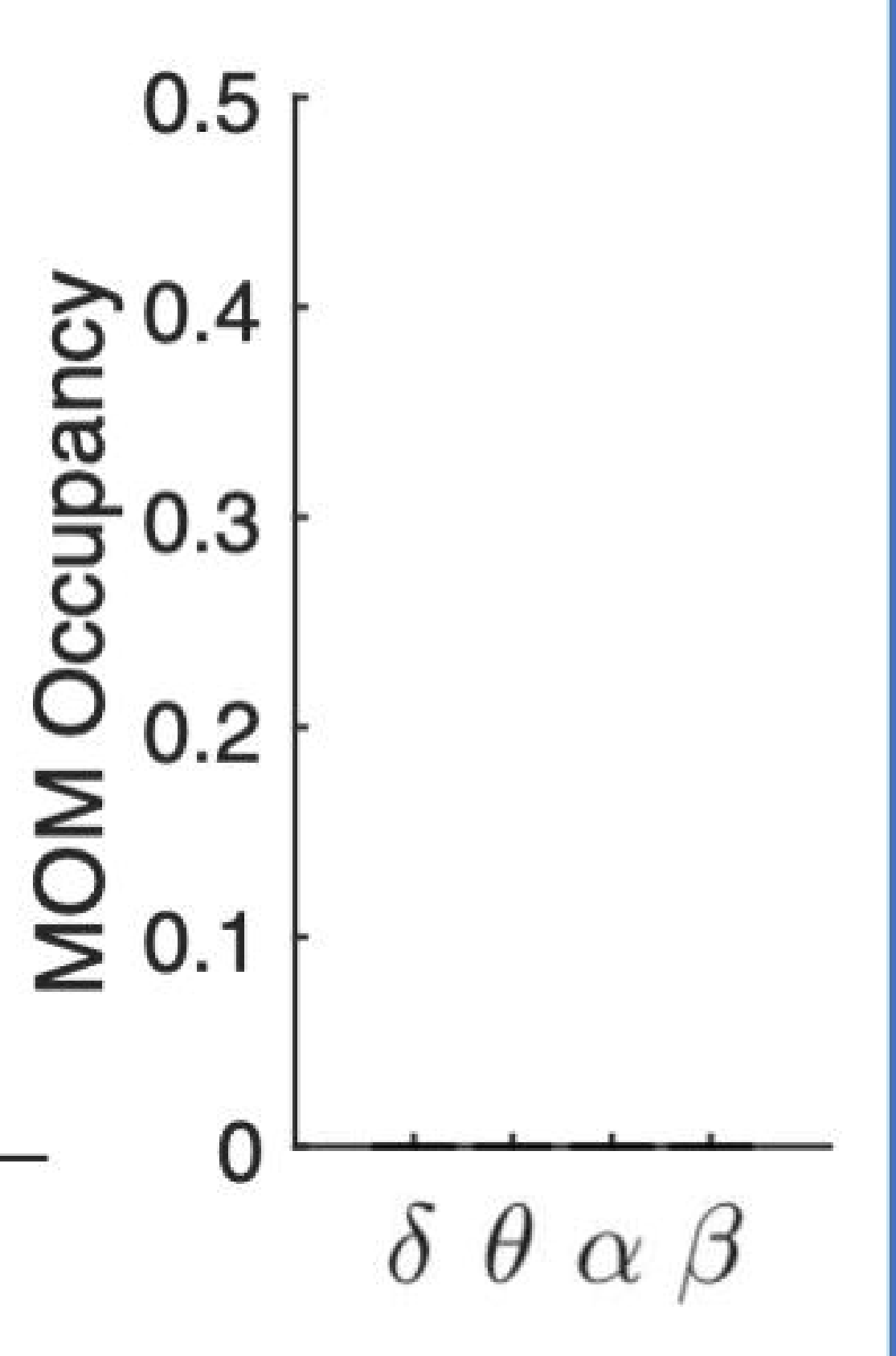
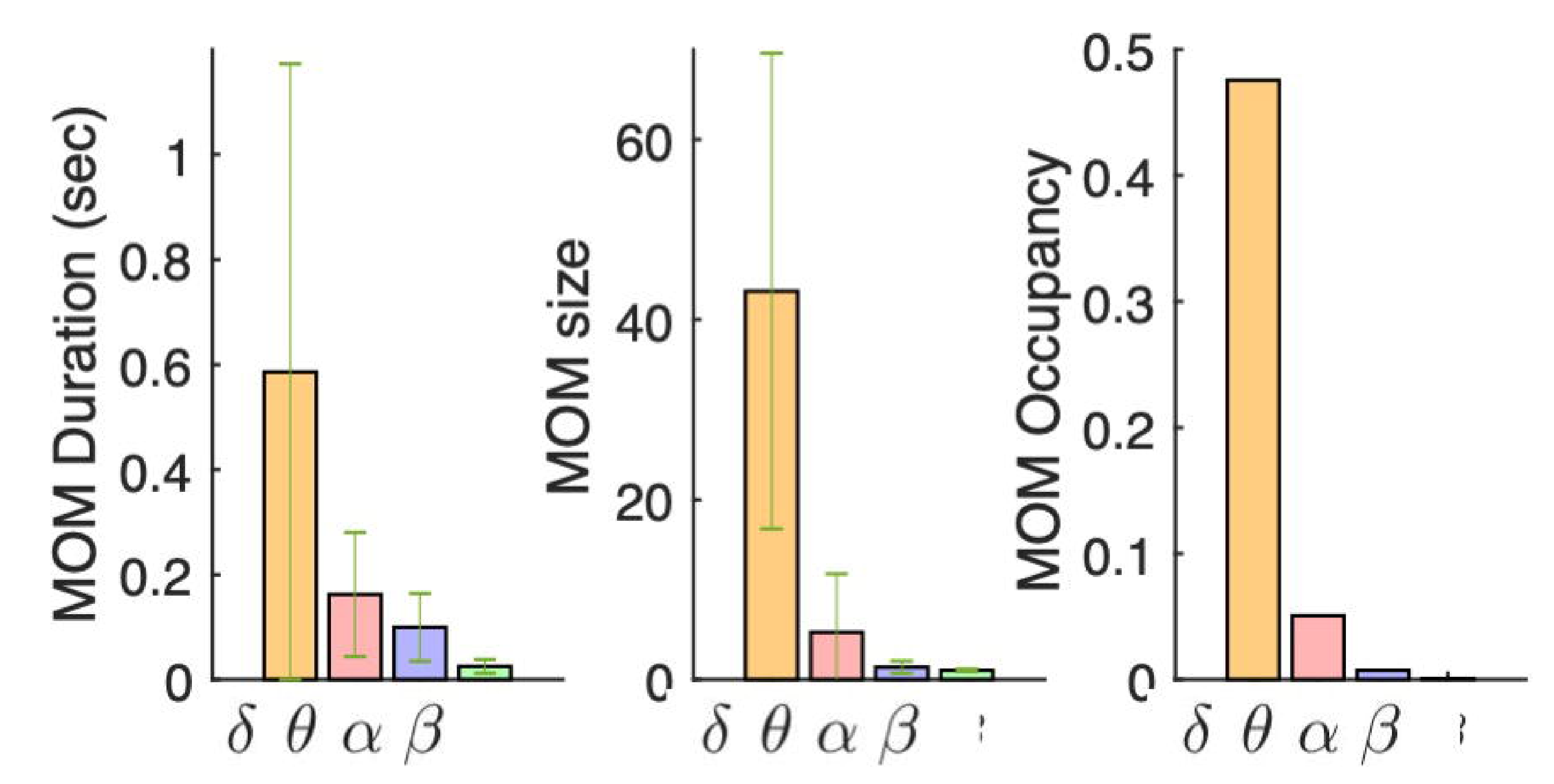

Optimal Range $\langle\tau\rangle=3 \mathrm{~ms} \mathrm{~K}=10$
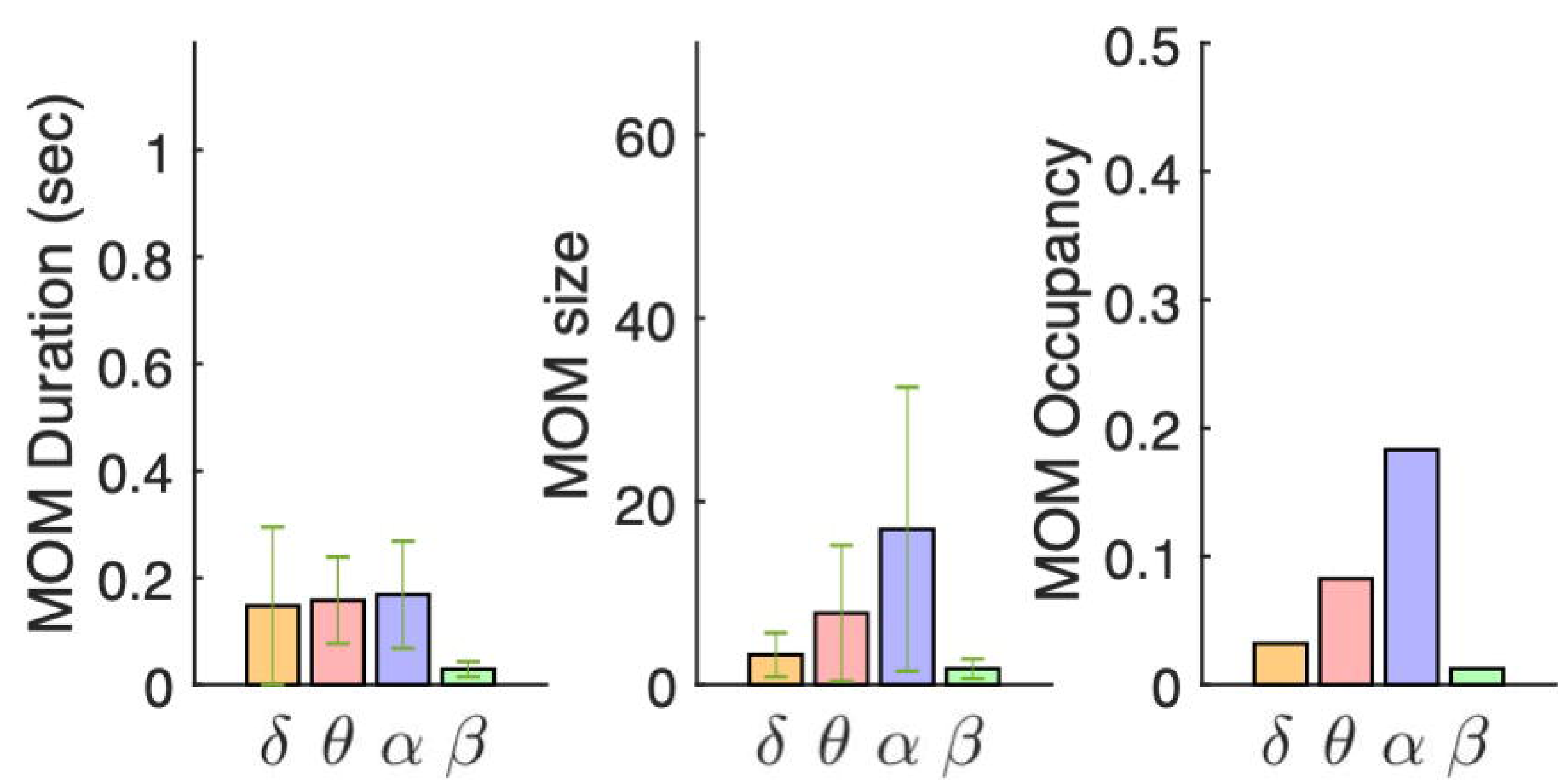

warms

Size

wrow

twing

nommon

Duration
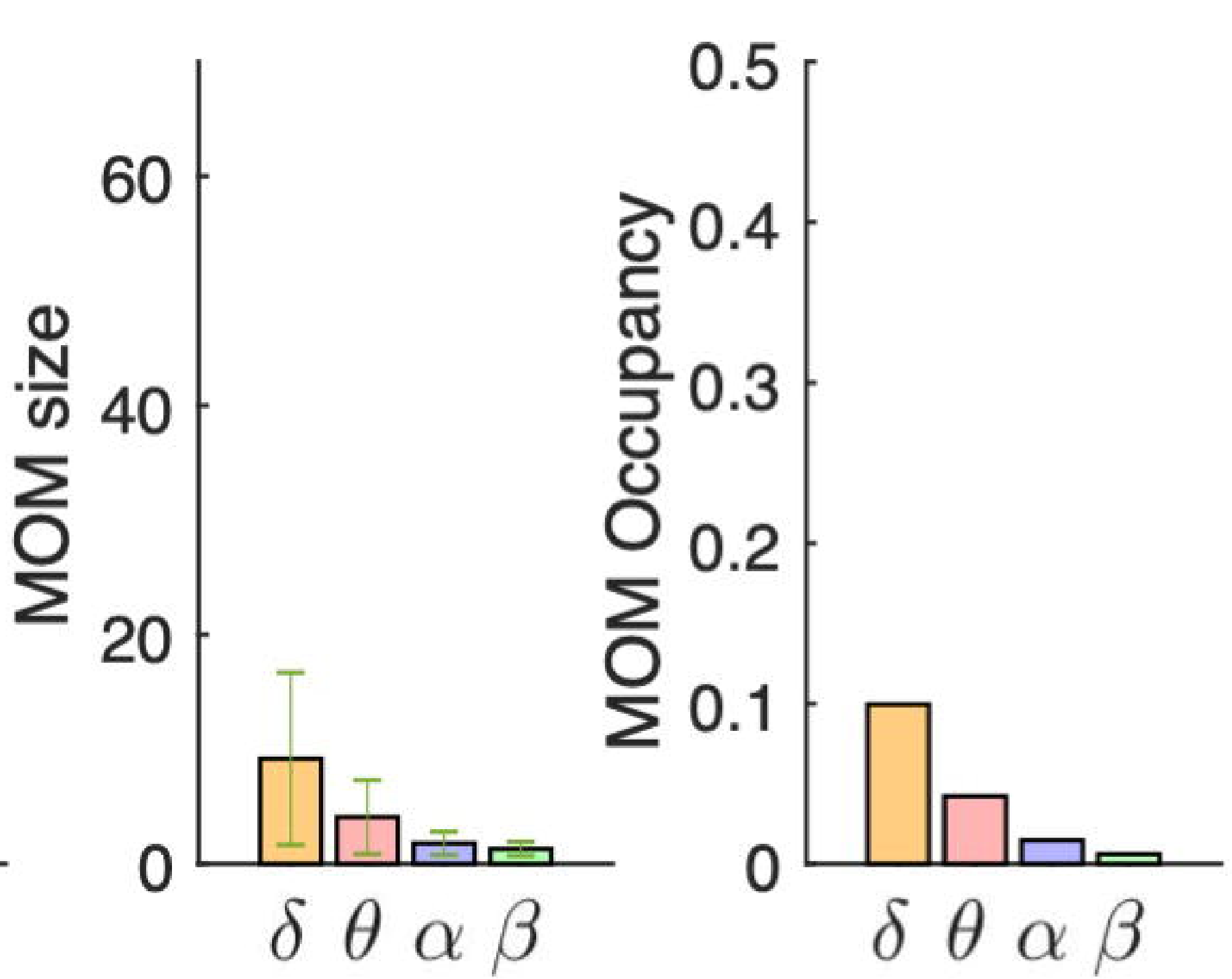

Weak Coupling $\langle\tau\rangle=3 \mathrm{~ms} \mathrm{~K}=0.1$

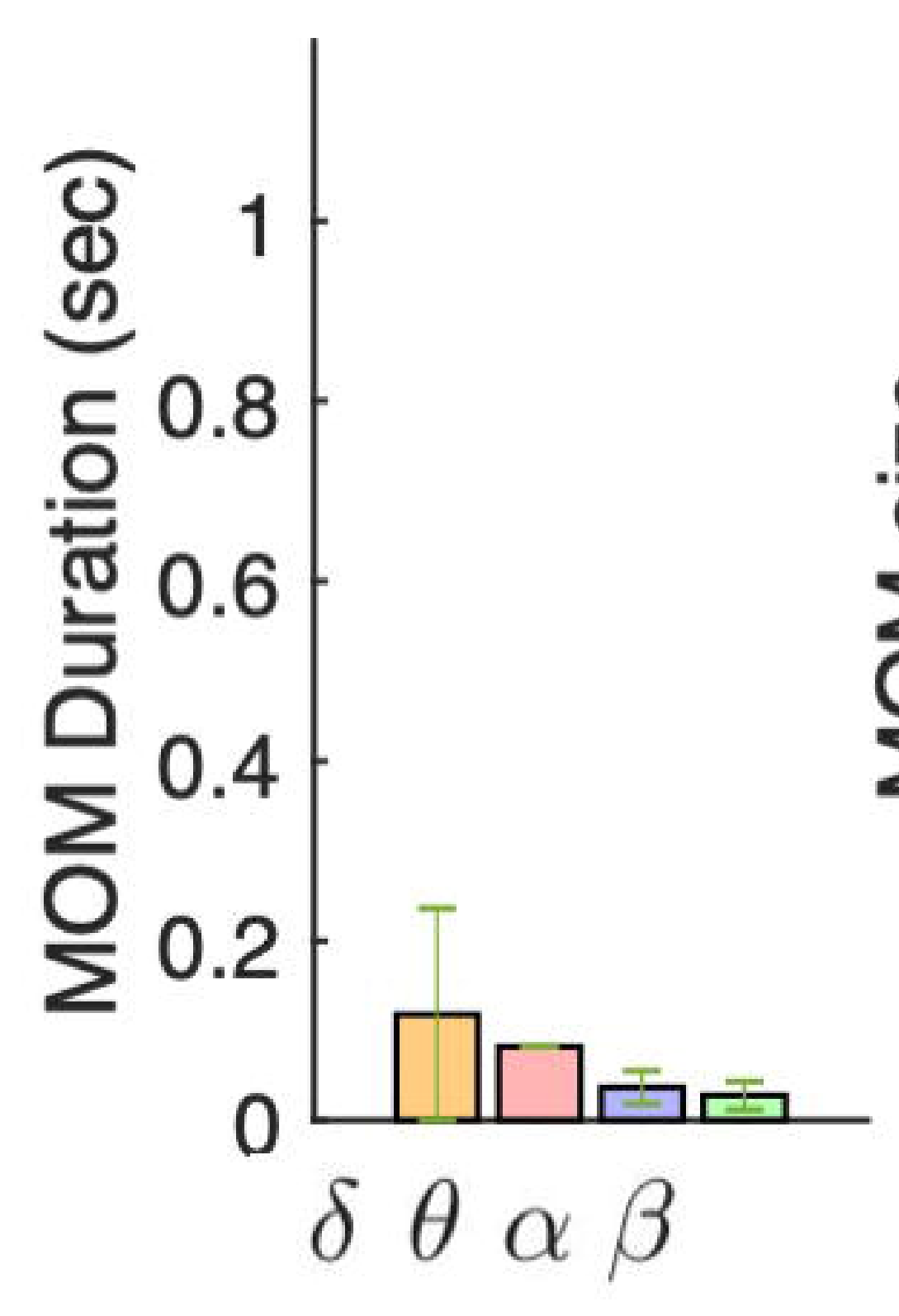

Conduction Delays

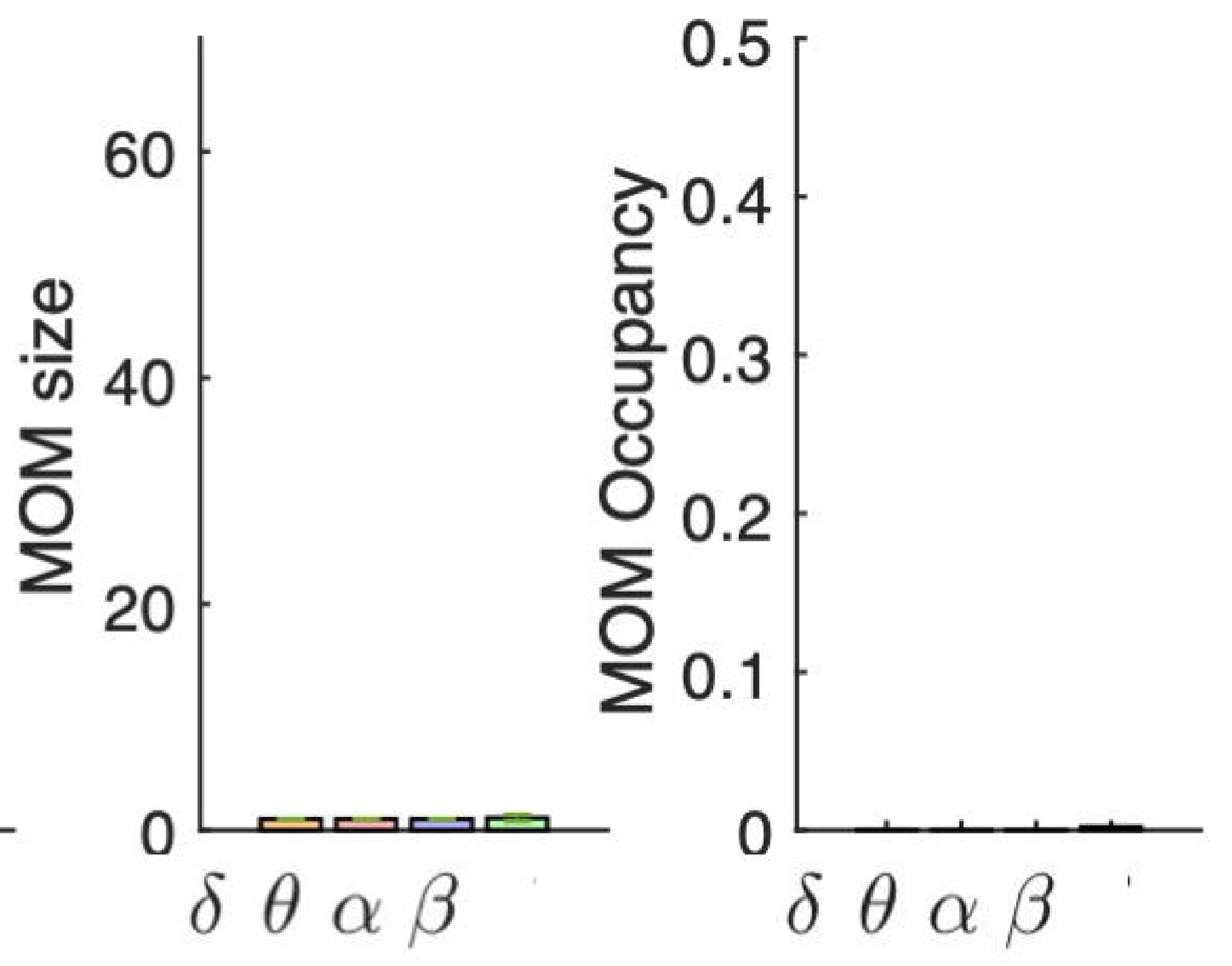




\section{Weak Coupling}

$\langle\tau\rangle=3 \mathrm{~ms} \mathrm{~K}=0.1$

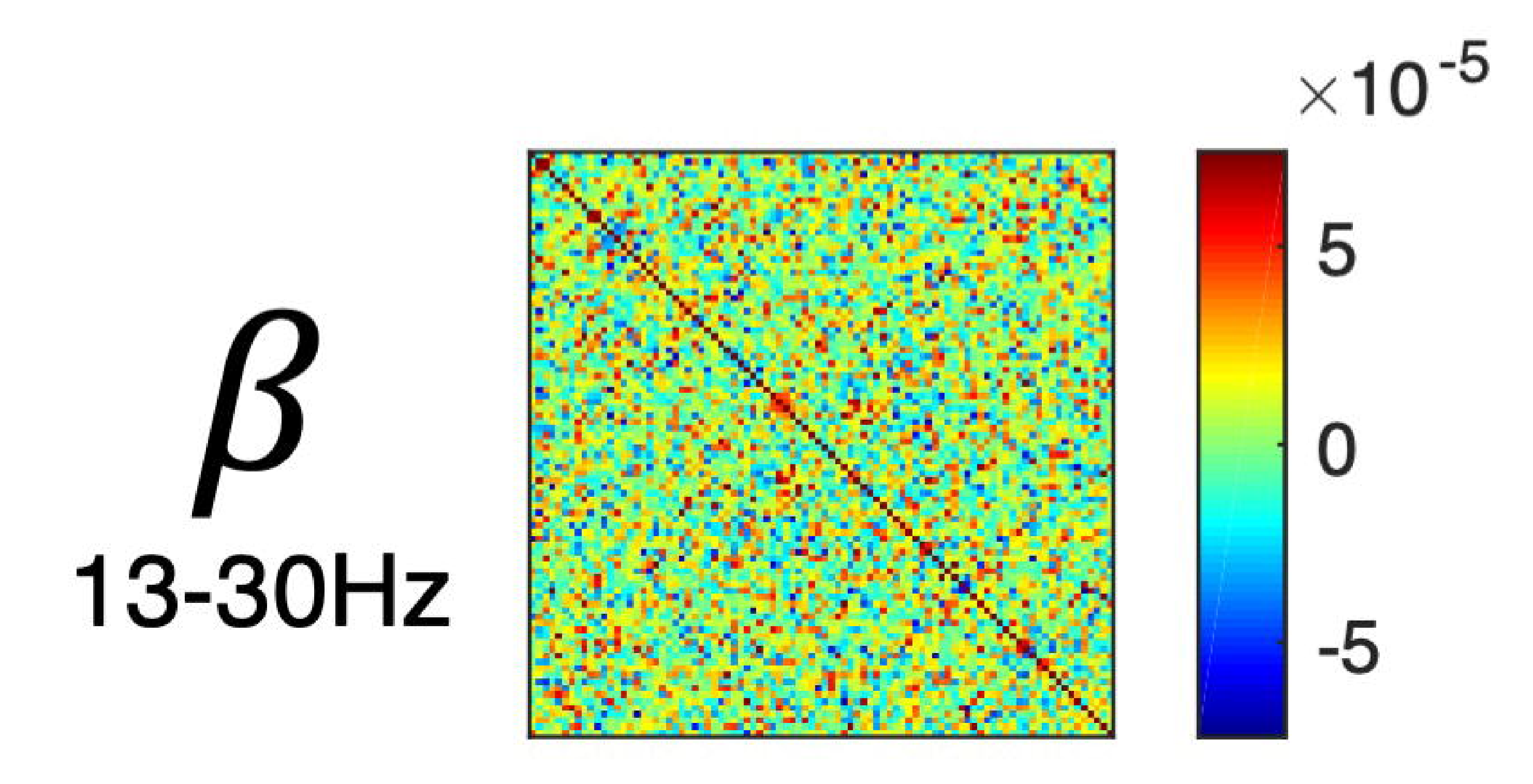

$\alpha$

$8-13 \mathrm{~Hz}$

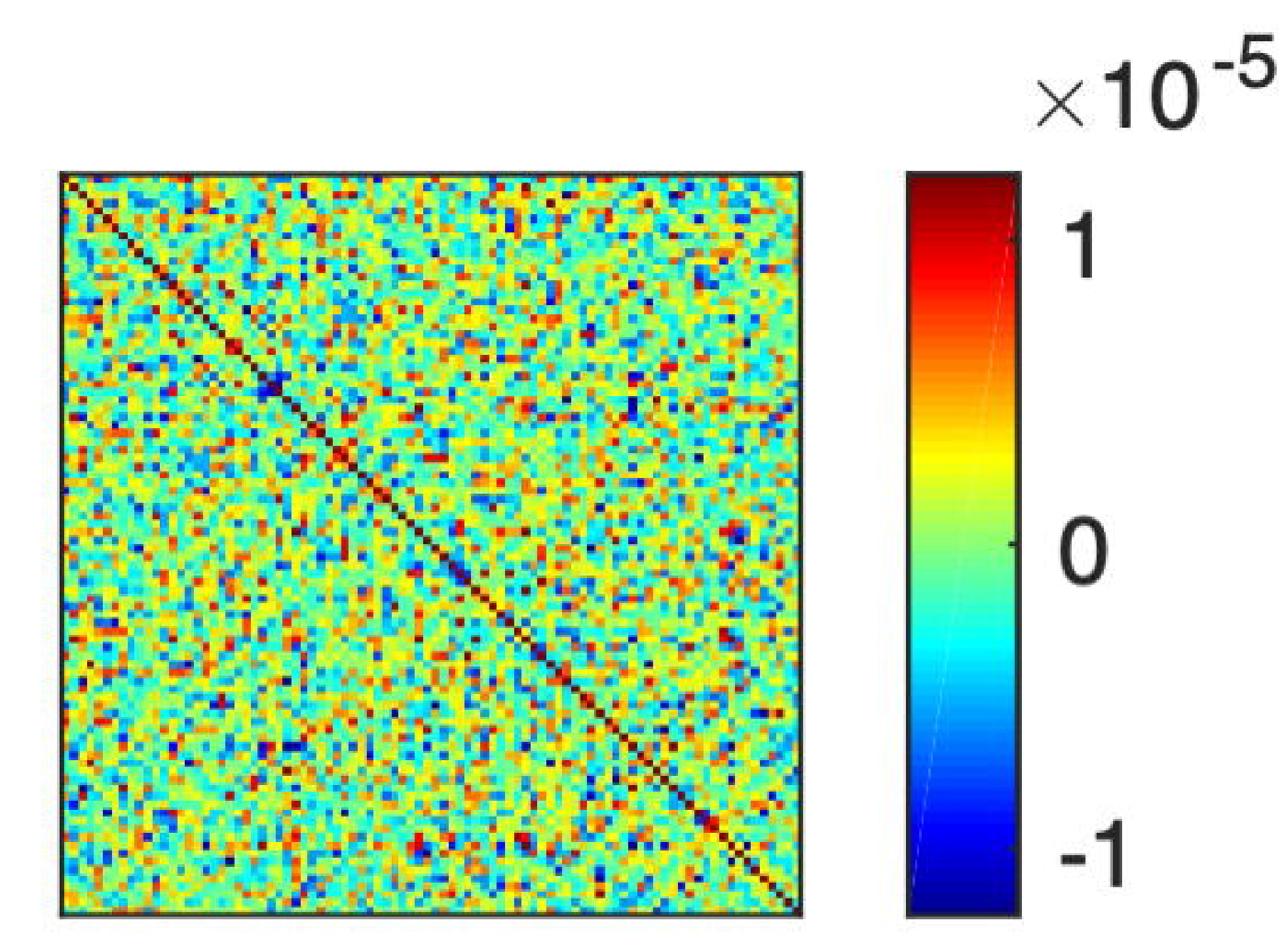

$\theta$

4-8Hz
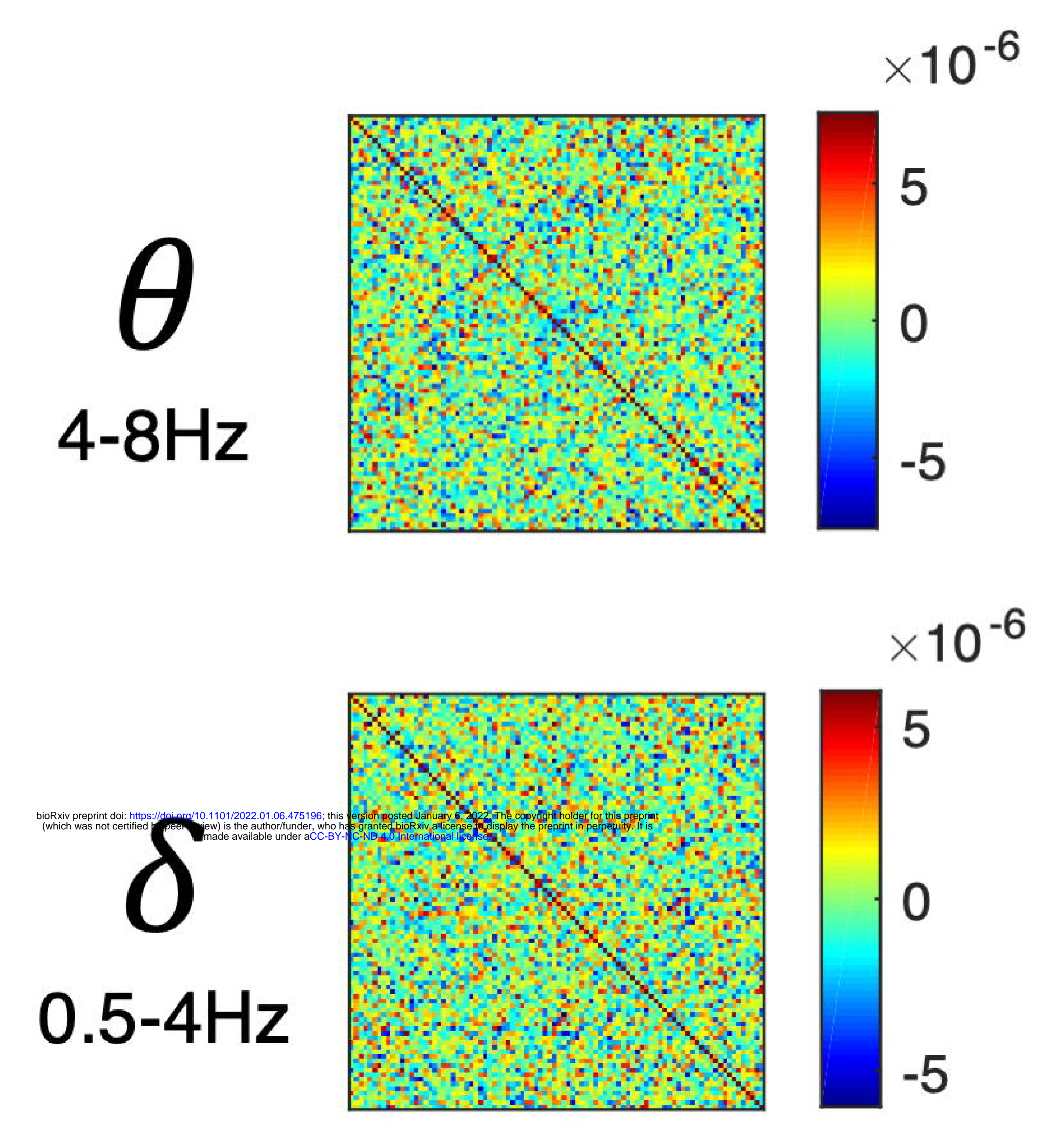

b

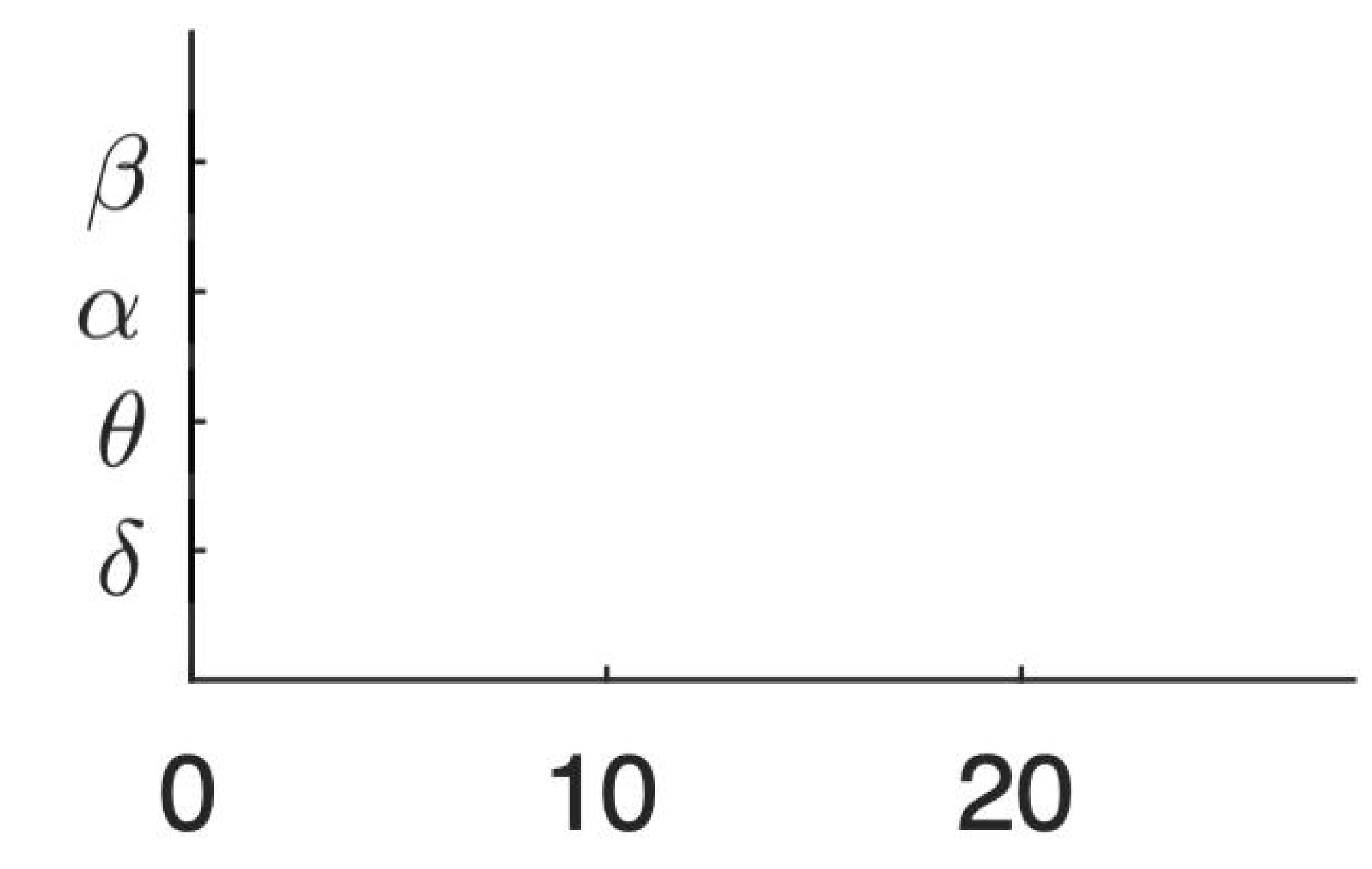

Eigenvalues $>$ baseline

\section{Optimal Range}

$\langle\tau\rangle=3 \mathrm{~ms} \mathrm{~K}=10$
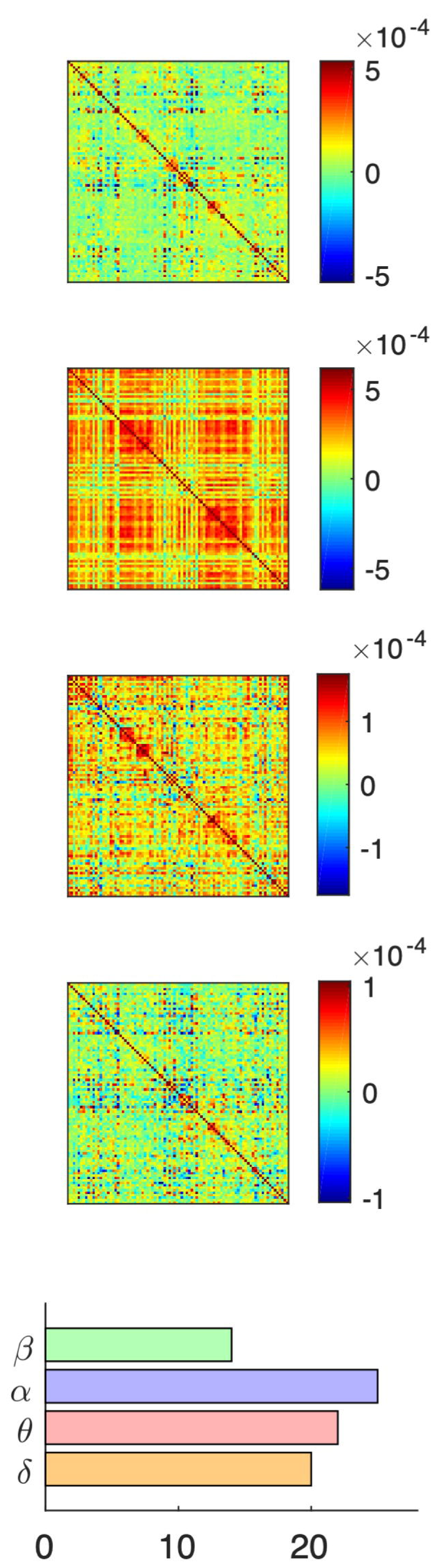

Eigenvalues $>$ baseline

\section{Strong Coupling}

$\langle\tau\rangle=3 \mathrm{~ms} \mathrm{~K}=50$
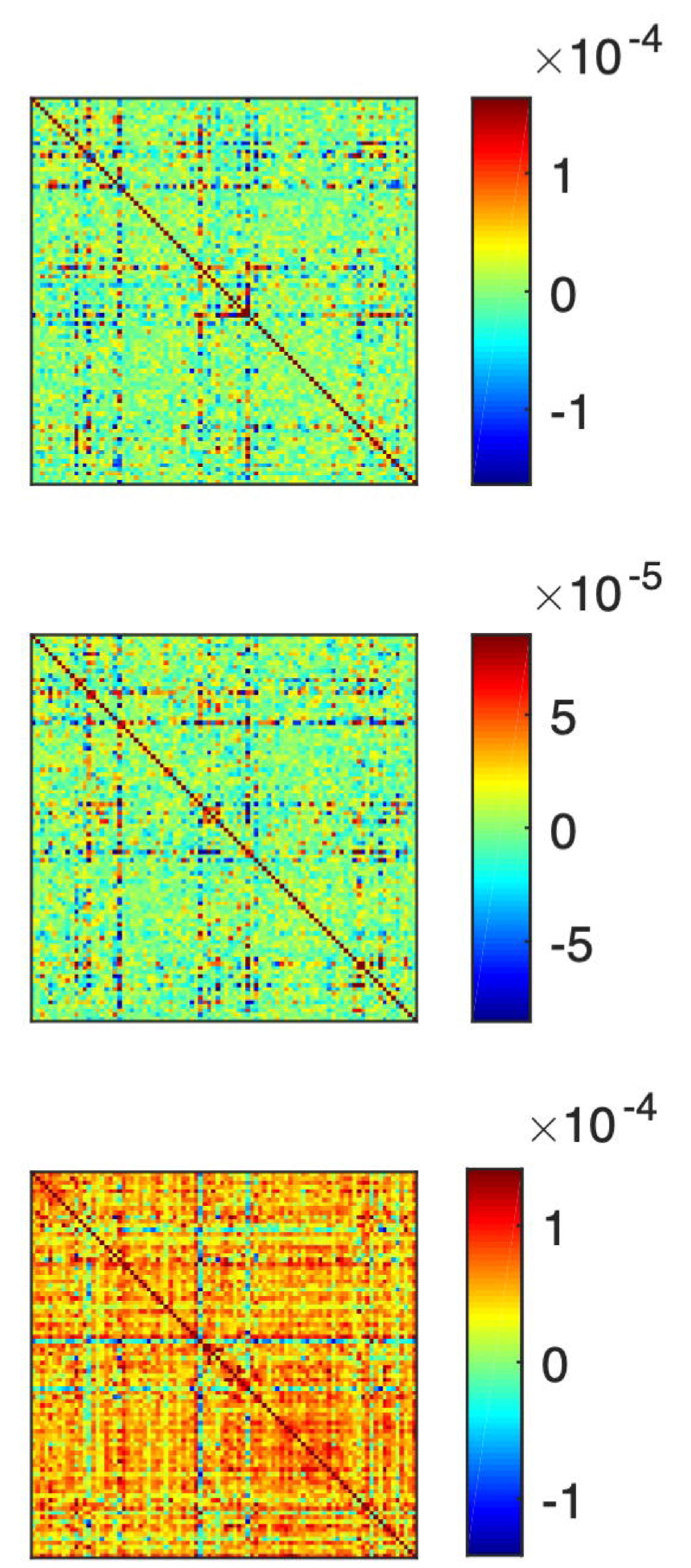

$\times 10^{-3}$
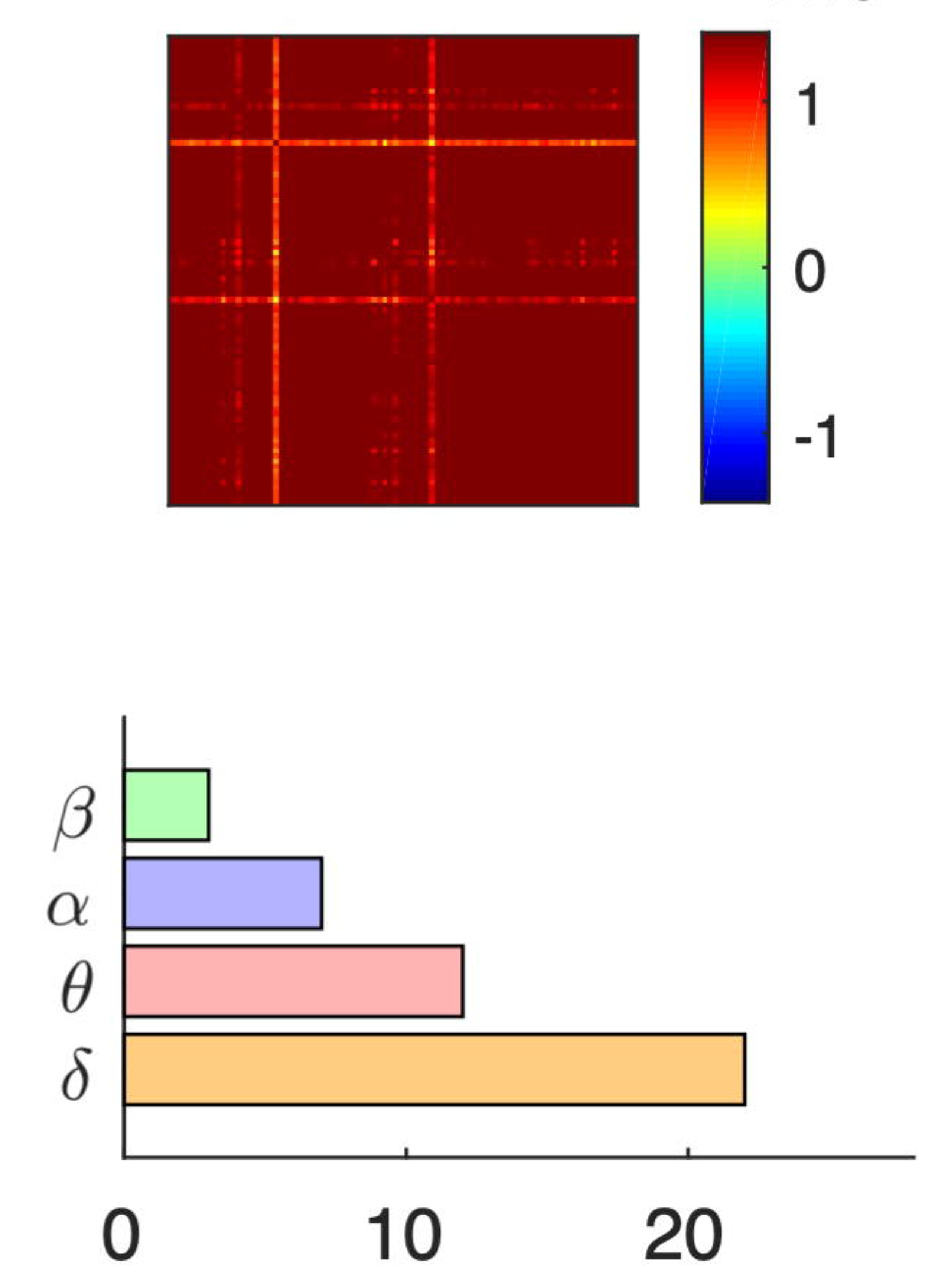

Eigenvalues $>$ baseline
Long Delays

$\langle\tau\rangle=10 \mathrm{~ms} \mathrm{~K}=10$
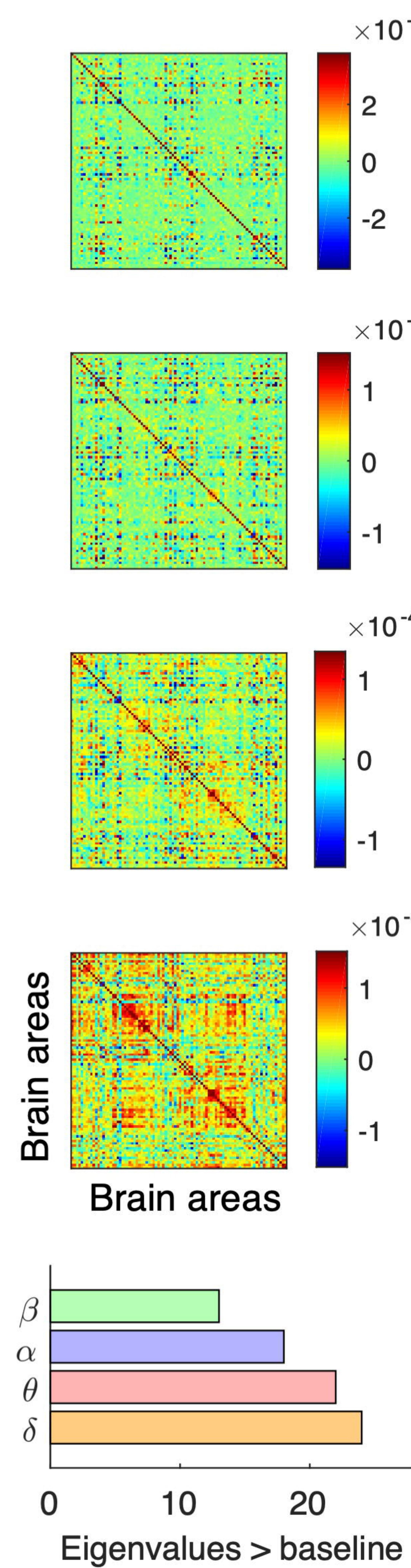